\title{
La eficacia de la prevención indicada de la depresión: una revisión
}

\author{
Fernando L. Vázquez ${ }^{1 *}$, Vanessa Blanco ${ }^{2}$, Ángela Torres ${ }^{3}$, Patricia Otero ${ }^{2}$ y Elisabet Hermida ${ }^{2}$ \\ ${ }^{1}$ Departamento de Psicologia Clinica y Psicobiología, Universidad de Santiago de Compostela \\ ${ }^{2}$ Unidad de Trastornos Depresivos, Universidad de Santiago de Compostela \\ ${ }^{3}$ Departamento de Psiquiatría, Radiología y Salud Pública, Universidad de Santiago de Compostela
}

\begin{abstract}
Resumen: En este trabajo se revisó la investigación acerca de la eficacia de las intervenciones psicológicas y psicoeducativas de prevención de la depresión en sujetos con síntomas depresivos, pero que no reunían los criterios para un episodio depresivo mayor actual (prevención indicada). Se seleccionaron 42 ensayos que evaluaron 36 programas de prevención encontrados en las bases de datos Medline, PsycINFO, ERIC, EMBASE, y en el registro de ensayos del Grupo Cochrane de Depresión, Ansiedad y Neurosis, en español e inglés, entre los años 1990 y 2013. La mayoría de los programas de prevención indicada analizados obtuvieron resultados positivos en la reducción de la sintomatología depresiva. Sin embargo, en general obtuvieron tamaños del efecto pequeños, y muchos de ellos no tuvieron efectos sobre la incidencia de depresión. Además, muchos de estos trabajos presentaban limitaciones metodológicas. Los datos señalados apoyan la necesidad de desarrollar intervenciones preventivas que sigan un modelo de prevención indicada, y que comprueben su eficacia a través de ensayos controlados aleatorios con las garantías metodológicas adecuadas.

Palabras clave: Prevención indicada; prevención secundaria; síntomas depresivos; depresión; terapia cognitivo conductual.
\end{abstract}

Title: The efficacy of indicated prevention of depression: A review. Abstract: This paper reviewed the research on the effectiveness of psychological and psychoeducational interventions to prevent depression in subjects with symptoms of depression, but that did not meet criteria for a current major depressive episode (indicated prevention). Forty-two randomized controlled trials, evaluating thirty-six preventive programs identified through the Medline, PsycINFO, ERIC and EMBASE databases and the Cochrane Collaboration of Depression, Anxiety and Neurosis Group Controlled Trials Register, in Spanish and English, between 1990 and 2013, were selected. Most indicated prevention programs showed positive effects on depressive symptoms. However, they have been found to produce small effect sizes, and many of them had no effect on the incidence of depressive disorders. In addition, many of these studies had methodological weaknesses. Current findings support the need to develop preventive interventions following one model of indicated prevention, and assessing their efficacy through randomized controlled trials with adequate methodological quality.

Key words: Indicated prevention; secondary prevention; depressive symptoms; depression; cognitive behavior therapy.

\section{Introducción}

La depresión es uno de los trastornos mentales más frecuentes, afectando a 340 millones de personas en el mundo (Organización Mundial de la Salud, 2004). En lo que a España se refiere, la prevalencia a los 12 meses en población adulta general oscila entre 1.300 .000 y 1.500 .000 personas, esto es, aproximadamente el 4\% de los mayores de 18 años de nuestro país (Gabilondo et al., 2010). Es la cuarta patología más importante en la estimación de carga de enfermedad a nivel mundial, la tercera en Europa y, teniendo en cuenta su ritmo de progresión, se prevé que será la segunda causa de carga de enfermedad en el mundo en el año 2030, sólo superada por el VIH/SIDA (Mathers y Loncar, 2006). Además, la depresión conlleva un elevado coste económico. En Europa, el coste total de la depresión se ha estimado en 118 billones de euros (5005 millones de euros anuales en España), debiéndose el 61\% de esta cifra a costes indirectos (Valladares, Dilla y Sacristán, 2009).

A pesar de esas graves repercusiones derivadas de la depresión, está infradiagnosticada e infratratada. Sin embargo, si se trata, la depresión tiene un buen pronóstico y responde bien a las medidas de apoyo. Las guías prácticas para el manejo de la depresión recomiendan la terapia cognitivo-conductual (TCC), la psicoterapia interpersonal (PTI) y distintos fármacos antidepresivos como los

\section{* Dirección para correspondencia [Correspondence address]:}

Fernando L. Vázquez. Departamento de Psicología Clínica y Psicobiología. Facultad de Psicología, Universidad de Santiago de Compostela. Campus Vida. 15782, Santiago de Compostela (España).

E-mail: fernandolino.vazquez@usc.es procedimientos de intervención más eficaces (p.e., Grupo de Trabajo sobre el Manejo de la Depresión Mayor en el Adulto, 2008; National Institute for Clinical Excellence, 2009). Más concretamente, en relación con el tratamiento psicológico, la proliferación de las terapias cognitivoconductuales ha generado un amplio rango de métodos de tratamiento altamente efectivos (Butler, Chapman, Forman y Beck, 2006; Oei y Dingle, 2008). Sin embargo, la elevada prevalencia de la depresión y la escasez de recursos asistenciales imposibilitan que todas las personas que la desarrollan puedan tener acceso a los recursos de tratamiento.

Las graves consecuencias del desarrollo de la depresión en jóvenes y adultos y las limitaciones del alcance del tratamiento subrayan la necesidad de implementar programas de prevención. De hecho, en los últimos años la prevención de la depresión ha despertado un enorme interés en el campo de estudio, especialmente en el desarrollo y evaluación de programas de prevención indicada. En el informe del Instituto de Medicina de EEUU se definió ese tipo de prevención como aquella que tiene como población objetivo a personas que ya han desarrollado signos y síntomas, pero que todavía no cumplen los criterios diagnósticos para un trastorno (Mrazek y Haggerty, 1994). Por lo tanto, la meta en los programas de prevención indicada para la depresión es "mantener a raya" los síntomas depresivos de manera que no traspasen la línea hacia un episodio depresivo mayor. El éxito de la intervención, viene definido por la reducción en la sintomatología depresiva $y$, fundamentalmente, por la reducción en el comienzo de nuevos episodios depresivos (incidencia). En los últimos 20 años, varios ensayos han tenido éxito en la reducción de la sintomatología depresiva y 
la incidencia de episodios depresivos (p.e., Clarke et al., 1995, 2001; Willemse, Smit, Cuijpers, y Tiemens, 2004). Estos estudios, realizados con distintas poblaciones y en distintos países, sugieren que la depresión se puede prevenir. A pesar de que existe algún trabajo de meta-análisis y revisión previo que ha comparado la eficacia de los programas de prevención de la depresión desde sus distintas perspectivas (universal, selectiva e indicada), éstos se han centrado fundamentalmente en adolescentes y mayores (p.e., Calear y Christensen, 2010; Forsman, Schierenbeck y Wahlbeck, 2011), pero no existen trabajos de revisión previos que hayan revisado de manera exhaustiva todos los ensayos controlados aleatorizados de prevención indicada de la depresión existentes, independientemente del tipo de población. Es más, en esas revisiones ya publicadas los aspectos analizados relacionados con la metodología y los resultados de los artículos incluidos en las mismas son muy limitados. En este trabajo se realizó una revisión exhaustiva de los estudios de prevención indicada de la depresión en personas de todas las edades realizados hasta la actualidad, de todas aquellas características sugeridos por la Cochrane (Higgins y Green, 2008), y se examinó su impacto sobre la sintomatología depresiva, la incidencia de la depresión mayor y otras variables.

\section{Método}

Se realizó una revisión sistemática de la literatura en las bases de datos electrónicas Medline, PsycINFO, ERIC, EMBASE, y en el registro de ensayos del Grupo Cochrane de Depresión, Ansiedad y Neurosis, en español e inglés, entre enero de 1990 y enero de 2013. Los términos utilizados para dicha búsqueda fueron prevención "Y" depresión, prevención indicada "Y" depresión, prevención "Y" sintomatología depresiva, prevención indicada "Y" sintomatología depresiva. Se delimitaron los resultados de la búsqueda con el término ensayo controlado aleatorizado. Además, se consultaron los artículos de revisión y metaanálisis publicados, y se revisó su bibliografía y la de los artículos originales para localizar nuevas investigaciones.

Se examinaron un total de 2146 resúmenes en Medline $(N=134)$; PsycInfo $(N=1810)$, ERIC $(N=23)$, EMBASE $(N=143)$ y en el registro de ensayos del citado grupo Cochrane $(N=16)$, así como 20 artículos de revisión y metaanálisis. De los 212 trabajos seleccionados para ser leídos en su totalidad tras la lectura de su resumen, 125 fueron excluidos tras su revisión completa tras verificarse que incumplían alguno de los criterios de inclusión. Para una descripción más detallada de la estrategia de búsqueda ver la Figura 1.

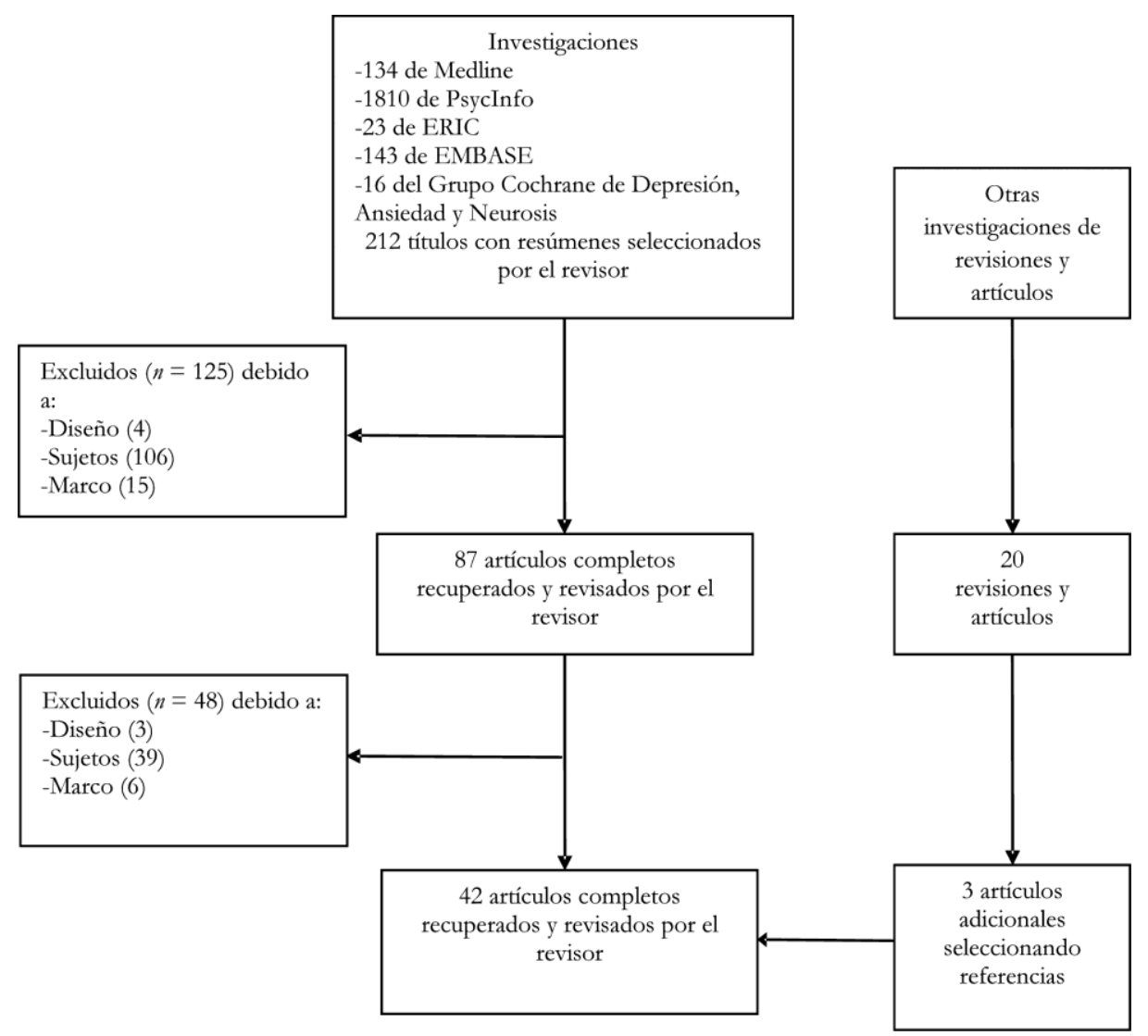

Figura 1. Estrategia de búsqueda. Artículos revisados, excluidos y seleccionados. 
Se incluyeron los ensayos controlados aleatorizados cuyo objetivo principal era la prevención indicada de la depresión, en los que se emplearon intervenciones psicológicas o psicoeducativas dirigidas a personas de todas la edades (niños, adolescentes, jóvenes y adultos) que presentaban sintomatología depresiva clínicamente significativa, pero no reunían los criterios para un trastorno depresivo mayor; y en los que compararon los datos de las intervenciones con los de un grupo placebo, con cualquier intervención de comparación o con la ausencia de intervención; y que evaluaron la presencia de sintomatología depresiva o de un episodio depresivo mayor a través de la utilización de instrumentos estandarizados. Para el establecimiento de la distinción entre intervenciones psicológicas y psicoeducativas se ha seguido el criterio establecido por Merry, McDowell, Hetrick, Bir y Muller (2008), que definieron las intervenciones como psicológicas cuando se enseñó a los participantes habilidades para reducir la depresión (p.e., manejo del estrés, reestructuración cognitiva, solución de problemas), y como psicoeducativas cuando se centraron solamente en proporcionar información, por ejemplo información sobre el modo de reconocer el trastorno depresivo y la manera de tratarlo. Se excluyeron de la revisión aquellos estudios donde se describían intervenciones de prevención indicada que no informaron de sus resultados, así como otros tipos de prevención.

De los estudios localizados en la búsqueda, 42 resultaron elegibles para esta revisión (6 de ellos informan de los resultados logrados en los seguimientos de estudios publicados previamente), siendo analizados los aspectos sugeridos por la Cochrane (Higgins y Green, 2008). A efectos descriptivos, un estudio y sus seguimientos fueron contabilizados como un único trabajo. En la Tabla 1 se presentan estos estudios ordenados alfabéticamente e indicando la identificación del trabajo (autores y año de publicación); la muestra utilizada (el número de sujetos, el porcentaje del sexo predominante, la media de edad y el porcentaje de abandonos); las intervenciones (los tipos de programas comparados y el número de sujetos en cada grupo); y los resultados obtenidos en la reducción de la sintomatología depresiva, la incidencia de depresión y otras variables clínicas.

\section{Resultados}

\section{Descripción de los Estudios}

La mayoría de las intervenciones incluidas en esta revisión fueron psicológicas (p.e., Allart-van Dam, Hosman, Hoogduin y Schaap, 2003, 2007; Le, Perry y Stuart, 2011; Seligman, Schulman y Tryon, 2007; Vázquez et al., 2012), excepto dos, que fueron psicoeducativas (Lara, Navarro y Navarrete, 2010; Liu et al., 2009).

En estas intervenciones, el factor de riesgo para desarrollar depresión considerado en 24 estudios (con sus correspondientes seguimientos) fue tener puntuaciones elevadas de depresión sin reunir los criterios diagnósticos para un episo- dio depresivo actual ${ }^{1}$ (Allart-van Dam et al., 2003, 2007; Clarke et al., 1995; Dobson, Hopkins, Fata, Scherrer y Allan, 2010; Dozeman et al., 2012; Gillham, Hamilton, Freres, Patton y Gallop, 2006; González, Fernández, Pérez y Amigo, 2006; Konnert, Dobson y Stelmach, 2009; Liu et al., 2009; Lynch, Tamburrino y Nagel, 1997; Mossey, Knott, Higgins y Talerico, 1996; Roberts, Kane, Thomson, Bishop y Hart, 2003; Roberts, Kane, Bishop, Matthews y Thomson, 2004; Seligman et al., 2007; Sheffield et al., 2006; Spence, Sheffield y Donovan, 2003; Stice, Burton, Bearman y Rohde, 2006; Stice, Rohde, Gau y Wade, 2010; Stice, Rohde, Seeley y Gau, 2008; Van Voorhees et al., 2008, 2009; Van't Veer-Tazelaar et al., 2009, 2011; Vázquez et al., 2012; Vázquez et al., 2013; Westerhof, Bohlmeijer, van Beljouw y Pot, 2010; Willemse et al., 2004; Young, Mufson y Davies, 2006; Young, Mufson y Gallop, 2010). Trece trabajos requirieron además criterios adicionales: presentar sintomatología depresiva elevada y/o un episodio depresivo mayor en remisión durante al menos dos meses en un estudio (Garber et al., 2009); estar en estado de gestación en cinco estudios (Lara et al., 2010; Le et al., 2011; Muñoz et al., 2007; Stamp, Williams y Crowther, 1995; Zlotnick, Johnson, Miller, Pearlstein y Howard, 2001), combinando una aproximación selectiva e indicada; estilo atribucional pesimista en dos estudios (Arnarson y Craighead, 2009, 2011; Seligman, Schulman, DeRubeis y Hollon, 1999); ser hijo de padres que habían recibido tratamiento por un trastorno depresivo en un estudio (Clarke et al., 2001); ser estudiante universitario (Vázquez et al., 2012); tener más de 18 años y síntomas de ansiedad de leves a moderados en un estudio (Fledderus, Bohlmeijer, Pieterse y Shreurs, 2012); ser mayor de 55 años en un estudio (Korte, Bohlmeijer, Cappeliez, Smit y Westerhof, 2012); y vivir en un área rural en un estudio (Puskar, Sereika y Tutsaie-Mumford, 2003).

$\mathrm{La}$ edad de los participantes en estas intervenciones fue variada, oscilando entre 11 (Gillham et al., 2006; Roberts et al., 2003, 2004; Young et al., 2006) y 91 años (Mossey et al., 1996) para aquellos trabajos que informaron del rango de edades.

El origen de los participantes fue EEUU en 17 estudios con sus seguimientos (Clarke et al., 1995, 2001; Garber et al., 2009; Gillham et al., 2006; Le et al., 2011; Lynch et al., 1997; Mossey et al., 1996; Muñoz et al., 2007; Puskar et al., 2003; Seligman et al., 1999, 2007; Stice et al., 2006, 2008, 2010; Van Voorhees et al., 2008, 2009; Young et al., 2006, 2010; Zlotnick et al., 2001); Holanda en siete estudios (Allart-van Dam et al., 2003, 2007; Dozeman et al., 2012; Korte et al., 2012; Fledderus et al., 2012; Van't Veer-Tazelaar et al., 2009, 2011; Westerhof et al., 2010; Willemse et al., 2004); Australia en cuatro estudios (Roberts et al., 2003, 2004; Sheffield et al.,

1 Allart van Dam et al. (2007), Arnarson y Craighead (2011), Roberts et al. (2004), Stice et al. (2010), Van Voorhees et al. (2009) y Van't Veer-Tazelaar et al. (2011) analizan los datos de seguimiento de Allart van Dam et al. (2003), Arnarson y Craighead (2009), Roberts et al. (2003), Stice et al. (2008), Van Voorhees et al. (2008) y Van't Veer-Tazelaar et al. (2009), respectivamente. 
2006; Spence et al., 2003; Stamp et al., 1995); España en tres estudios (González et al., 2006; Vázquez et al., 2012, 2013); Canadá en dos estudios (Dobson et al., 2010; Konnert et al., 2009); China en un estudio (Liu et al., 2009); México en un estudio (Lara et al., 2010); e Islandia en un estudio (Arnarson y Craighead, 2009, 2011).

Se utilizaron distintos tipos de programas de prevención. Diez estudios incluyeron el Curso de Afrontamiento de la Depresion (Coping With Depression [CWD]) ${ }^{2}$, original o adaptado (Allart-van Dam et al., 2003, 2007; Clarke et al., 1995, 2001; Dobson et al., 2010; Konnert et al., 2009; Le et al., 2011; Muñoz et al., 2007; Van't Veer-Tazelaar et al., 2009, 2011; Vázquez et al., 2012; Willemse et al., 2004); cuatro estudios emplearon Psicoterapia Interpersonal (Interpersonal Psychotherapy [IPT]) ${ }^{3}$ (Mossey et al., 1996; Young et al., 2006, 2010; Zlotnick et al., 2001); dos de los estudios incluyeron programas elaborados por la Universidad de Pensilvania, como el Programa de Resistencia a la Adversidad de Pensilvania (Penn Resiliency Program [PRP]) (Gillham et al., 2006; Roberts et al., 2003, 2004); tres estudios emplearon Terapia de Solución de Problemas (Problem Solving Therapy [PST]) ${ }^{5}$ (Lynch et al., 1997; Spence et al., 2003; Vázquez et al., 2013); un estudio empleó el programa Enseñar a los Niños a Solucionar (Teaching Kids to Cope [TKC] $)^{6}$ (TKC; Puskar et al., 2003); un estudio empleó el programa Salud Mental de Mamás y Bebés' (Lara et al., 2010); un estudio empleó el programa de biblioterapia Mind Over

\footnotetext{
${ }^{2}$ El CWD, diseñado por Lewinsohn y Clarke (1984), es una intervención de prevención cognitivo-conductual adaptada de una intervención originalmente diseñada como tratamiento para la depresión mayor. Incluye los siguientes componentes: información sobre la depresión y la perspectiva cognitivoconductual de la depresión; técnicas de relajación; estrategias para incrementar las actividades agradables; habilidades cognitivas; habilidades sociales y asertividad; y procedimientos de prevención de recaídas. Otro programa de prevención que se originó en esa misma terapia para la depresión es el Curso de Prevención de la Depresión diseñado por Muñoz et al. (2007).

${ }^{3}$ La ITP asume que los síntomas de la depresión ocurren en un contexto psicosocial e interpersonal. Se focaliza en psicoeducación en relación con la depresión y la prevención, y construcción de habilidades focalizada en disputas de rol interpersonales, transiciones de rol y déficits interpersonales.

${ }^{4}$ El PRP es un programa basado en la terapia cognitivo-conductual que enseña a los participantes la conexión entre acontecimientos vitales, sus creencias sobre esos eventos, y las consecuencias emocionales de sus interpretaciones.

${ }^{5}$ La PST es una estrategia cognitivo-conductual que incluye el entrenamiento de una serie de habilidades: orientación hacia el problema, definición y formulación del problema, generación de alternativas, toma de decisiones y puesta en práctica de la solución y verificación.

${ }^{6} \mathrm{El} \mathrm{TKC}$ es un programa cognitivo-conductual que aborda una serie de actividades diseñadas para mejorar el afrontamiento de acontecimientos vitales estresantes y disminuir la sintomatología depresiva, a través de técnicas de detección y pruebas de realidad de los pensamientos distorsionados, exploración y práctica de la identificación de problemas, y formas alternativas de enfocar una situación y de responder a ella.

${ }^{7}$ Este programa incluye educación sobre la depresión posparto y factores de riesgo, estrategias para reducir los niveles de depresión (incrementar el pensamiento positivo y las actividades agradables, mejorar la autoestima, incrementar el autocuidado), así como un componente grupal.
}

Mood ${ }^{8}$ (Liu et al., 2009); un estudio empleó terapia de apoyo y expresión (Stamp et al., 1995); dos estudios utilizaron un programa de terapia de revisión de vida ${ }^{9}$ (Korte et al., 2012; Westerhof et al., 2010); un estudio y su correspondiente seguimiento emplean el programa de Transición a la Adultez. Competente con Entrenamiento Cognitivo-Conductual e Interpersonal (Competent Adulthood Transition With Cognitive-behavioral and Interpersonal Training [CATCH-IT] ${ }^{10}$ (Van Voorhees et al., 2008, 2009); un estudio empleó terapia de aceptación y compromiso (Fledderus et al., 2012); un estudio empleó una aproximación escalonada con observación, programación de actividades, revisión de vida y consulta con el médico general (Dozeman et al., 2012); mientras que otros ocho estudios no son tan concretos y simplemente especifican que se fundamen$\tan$ en componentes cognitivo-conductuales (Garber et al., 2009; Sheffield et al., 2006; Seligman et al., 1999, 2007; Stice et al., 2006, 2008, 2010), técnicas cognitivo-conductuales junto con potenciación de recursos personales y apoyo social (González et al., 2006); o cognitivo-conductuales y evolutivos (Arnarson y Craighead, 2009, 2011).

Las condiciones de comparación fueron diversas. Veinte estudios compararon el programa de prevención con una condición de atención habitual (Allart-van Dam et al., 2003, 2007; Arnarson y Craighead, 2009, 2011; Clarke et al., 1995, 2001; Dozeman et al., 2012 Garber et al., 2009; Gillham et al., 2006; Konnert et al., 2009; Korte et al., 2012; Lara et al., 2010; Le et al., 2011; Mossey et al., 1996; Puskar et al., 2003; Roberts et al., 2003, 2004; Stamp et al., 1995; Van't VeerTazelaar et al., 2009, 2011; Vázquez et al., 2013; Willemse et al., 2004; Young et al., 2010; Zlotnick et al., 2001); seis con una condición de atención activa y/o placebo (Dobson et al., 2010; Muñoz et al., 2007; Van Voorhees et al., 2008, 2009; Vázquez et al., 2012; Westerhof et al., 2010; Young et al., 2006); cuatro con una condición de no intervención (Lynch et al., 1997; Seligman et al., 1999, 2007; Spence et al., 2003); dos con una condición de lista de espera (González et al., 2006; Liu et al., 2009). En Sheffield et al. (2006) la condición de intervención se comparó con una condición de no intervención y con la misma condición de intervención en distintos tipos de muestra. En Stice et al. (2006, 2008, 2010) y Fledderus et al. (2012) se compararon los resultados de la intervención con otras condiciones de intervención, y con condiciones de control lista de espera o con intervenciones mínimas.

El número de sesiones estuvo comprendido entre 3 (Stamp et al., 1995) y 15 sesiones (Clarke et al., 1995, 2001;

\footnotetext{
${ }^{8}$ Se trata de un manual de autoayuda con un enfoque cognitivo, que aborda la modificación de los pensamientos distorsionados por otros más adaptativos.

9 Esta terapia está basada en el esfuerzo intencionado para la recuperación de recuerdos autobiográficos.

${ }^{10}$ El CATCH-IT incluye 14 módulos basados en la psicoterapia cognitivoconductual y de activación conductual, psicoterapia interpersonal, y un concepto de resiliencia comunitaria, centrándose en el aprendizaje de estrategias para abordar dificultades relacionadas con la depresión e incrementar conductas protectoras contra la misma.
} 
Dobson et al., 2010), con duraciones comprendidas entre 510 minutos (Van Voorhees et al., 2008, 2009) y 2 horas (Lara et al., 2010; Westerhof et al., 2010) para los estudios que informaron de la duración de las sesiones.

Los programas fueron aplicados por psicólogos (incluyendo psicólogos escolares) en 17 estudios (Allart-van Dam et al., 2003, 2007; Arnarson y Craighead, 2009, 2011; Clarke et al., 1995, 2001; Dobson et al., 2010; Fledderus et al., 2012; Garber et al., 2009; Konnert et al., 2009; Le et al., 2011; Lynch et al., 1997; Muñoz et al., 2007; Seligman et al., 1999, 2007; Stice et al., 2006, 2008, 2010; Vázquez et al., 2012, 2013); por profesionales de la salud en siete estudios (Lara et al., 2010; Mossey et al., 1996; Puskar et al., 2003; Stamp et al., 1995; Van Voorhees et al., 2008, 2009; Westerhof et al.,
2010; Van’t Veer-Tazelaar, 2009, 2011); psicólogos en colaboración con profesionales de la salud en tres estudios (González et al., 2006; Roberts et al., 2003, 2004; Sheffield et al., 2006); o con profesionales de áreas sociales en tres estudios (Gillham et al., 2006; Young et al., 2006, 2010); por personal de centros de la tercera edad, enfermeras de salud mental y médicos de cabecera en un estudio (Dozeman et al., 2012); por terapeutas en servicios de salud mental en un estudio (Korte et al., 2012); y por profesionales o paraprofesionales de áreas educativas en un estudio (Spence et al., 2003). En dos estudios no se informó de esta cuestión (Willemse et al., 2004; Zlotnick et al., 2001). En un estudio de biblioterapia (Liu et al., 2009), no existía facilitador, sino que el propio sujeto adecuaba sus lecturas a sus necesidades.

Tabla 1. Ensayos controlados aleatorios de prevención indicada de la depresión.

\begin{tabular}{lccc}
\hline $\begin{array}{l}\text { Publica- } \\
\text { ción }\end{array}$ & Muestra & Intervenciones & Resultados \\
\end{tabular}
de edad, pérdida de sujetos)

Allart-van 110 adultos de 18-65 años $\quad$ GE: Curso de Afrontamiento de la - GE mostró efectos beneficiosos sobre la sintoDam et al. $61.8 \%$ mujeres Depresión $(n=68) \quad$ matología depresiva, actividades agradables, autoes-

(2003) $\quad$ Edad media $=45.5(D T=9.9)$ $25.0 \%$

GC: Atención habitual $(n=42)$ tima, habilidades sociales, apoyo social, y pensamientos depresivos.

-Cambios en cogniciones depresivas y autoestima mediadores de los síntomas depresivos.

\begin{tabular}{llll}
\hline $\begin{array}{l}\text { Allart-van } \\
\text { Dam et al. } \\
(2007)^{\mathrm{a}}\end{array}$ & $\begin{array}{l}110 \text { adultos de 18-65 años } \\
\begin{array}{l}\text { Edad media }=45.5(D T=9.9) \\
25.0 \%\end{array}\end{array}$ & $\begin{array}{l}\text { GE: Curso de Afrontamiento de la } \\
\text { Depresión }(n=68)\end{array}$ & $\begin{array}{l}\text {-No se encontraron diferencias significativas en la } \\
\text { incidencia de trastornos depresivos entre GE y GC } \\
\text { en seg. } 12 \text { meses. }\end{array}$ \\
& GC: Atención habitual $(n=42)$ & $\begin{array}{l}\text {-Sólo participantes con sintomatología inicial baja } \\
\text { se beneficiaron a largo plazo. }\end{array}$
\end{tabular}

Arnarson y 171 jóvenes de 4-15 años $\quad$ GE: Intervención psicológica $(n=$-GE tasa significativamente más baja de depresión Craighead $55.6 \%$ mujeres GE; $51.1 \%$ hombres 81$) \quad$ y distimia que GC en el postest $(\mathrm{GE}=0 \%$, GC $=$ (2009) GC

Edad media $=$ n.d.

GC: Atención habitual $(n=90)$ $2.5 \%)$ y en el seg. 6 meses $(\mathrm{GE}=1.6 \%, \mathrm{GC}=$ $12.9 \%$ post.; $20.5 \%$ seg. 6 meses

Arnarson y 171 jóvenes de $14-15$ años

Craighead $\quad 55.6 \%$ mujeres GE; $51.1 \%$ hombres 81 )

(2011) $\quad$ GC

Edad media $=$ n.d.

$12.9 \%$ post.; $20.5 \%$ seg. 6 meses

GE: Intervención psicológica $(n=$-Los efectos preventivos se mantuvieron en el seguimiento a los 12 meses.

-Dos de los participantes del GE manifestaron un episodio depresivo mayor, frente 13 del GC.

-El nivel inicial de síntomas depresivos predijo el primer episodio de depresión y/o distimia entre los sujetos del GC.

Clarke et al. 150 adolescentes de $9^{\circ}$ y $10^{\circ}$ grado $\quad$ GE: Curso de Afrontamiento del -GE tasa significativamente más baja de incidencia

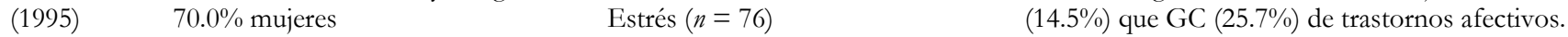

Edad media $=15.3(D T=0.7)$

$26.7 \%$ seg. 12 meses

GC: Atención habitual $(n=74)$

Clarke et al. 94 adolescentes de 13 a 18 años GE: Curso de Afrontamiento del -GE sintomatología depresiva significativamente (2001) $\quad 59.6 \%$ mujeres Estrés para Adolescentes (abrevia- menor que GC.

Edad media: $\mathrm{GE}=14.4(D T=1.4) ;$ do $)(n=45)$

$\mathrm{GC}=14.7(D T=1.5)$

$33.0 \%$ seg. 24 meses.

- Incidencia de depresión mayor a los 15 meses

GC: Atención habitual $(n=49)$ más baja en GE $(9.3 \%)$ que en GC $(28.8 \%)$.

Dobson et al. 46 estudiantes de 13-18 años GE: Curso de Afrontamiento del -GE y GC mostraron mejorías significativas en la sintomatología depresiva y la autoestima a lo largo Edad media: $\mathrm{GE}=15.1(D T=1.1)$;

$\mathrm{GC}=15.5(D T=1.1) \quad$ GC: Let's Talk $(n=21)$ $39.1 \%$ del tiempo.

-No se encontraron diferencias significativas entre los dos grupos.

Dozeman et 185 residentes en centros para per- GE: Programa de atención escalo- -El programa de atención escalonada es eficaz en la al. (2012) sonas mayores nada $(n=93)$ reducción de la incidencia de depresión, pero no en GE $72 \%$ mujeres; GC $73.9 \%$ mujela prevención del inicio de trastornos de ansiedad. 


\begin{tabular}{|c|c|c|c|}
\hline & $\begin{array}{l}\text { res } \\
\text { Edad media: } \mathrm{GE}=84.5(D T= \\
6.7) ; \mathrm{GC}=84.2(D T=6.4) \\
30 \%\end{array}$ & GC: Atención habitual $(n=92)$ & \\
\hline $\begin{array}{l}\text { Fledderus et } \\
\text { al. (2012) }\end{array}$ & $\begin{array}{l}376 \text { adultos de la población general } \\
\text { GE1 } 69.6 \% \text { mujeres; GE2 } 69.6 \% \\
\text { mujeres; GC } 69.8 \% \text { mujeres } \\
\text { Edad media: GE1 }=42.6(D T= \\
\text { 10.9); GE2 }=42.3(D T=11.1) ; \mathrm{GC} \\
=42.5(D T=11.3) \\
9.6 \% \text { post, } 18.4 \% \text { seg. }\end{array}$ & $\begin{array}{l}\text { GE1: Programa de autoayuda con } \\
\text { apoyo extensivo por email }(n= \\
\text { 125) } \\
\text { GE2: Programa de autoayuda con } \\
\text { apoyo mínimo por email }(n=125) \\
\text { GC: Lista de espera } \\
(n=126)\end{array}$ & $\begin{array}{l}\text {-En las condiciones experimentales se encontraron } \\
\text { reducciones significativas en depresión, ansiedad, } \\
\text { fatiga, evitación de experiencias y mejoras en salud } \\
\text { mental positiva y conciencia plena, sin diferencias } \\
\text { entre ambas. } \\
\text {-Los efectos se mantuvieron en seg. } 3 \text { meses }\end{array}$ \\
\hline $\begin{array}{l}\text { Garber et al. } \\
(2009)\end{array}$ & $\begin{array}{l}316 \text { jóvenes de } 13-17 \text { años } \\
58.5 \% \text { mujeres } \\
\text { Edad media }=14.8(D T=1.4) \\
4.8 \% \text { post.; } 9.5 \% \text { seg. }\end{array}$ & $\begin{array}{l}\text { GE: Programa de prevención cog- } \\
\text { nitivo-conductual }(n=159) \\
\text { GC: Atención habitual }(n=157)\end{array}$ & $\begin{array}{l}\text {-Incidencia de episodios depresivos más baja en el } \\
\text { GE que en el GC en el seg. a los } 6 \text { meses }(21.4 \% \\
\text { vs. } 32.7 \%) \text {. }\end{array}$ \\
\hline
\end{tabular}

\begin{tabular}{lll}
\hline Gillham et al. & 271 niños de $11-12$ años & GE: Penn Resiliency Program $(n$ \\
(2006) & $53.1 \%$ mujeres & 147) \\
& Edad media $=$ n.d. & GC: Atención habitual $(n=124)$ \\
& $20.1 \%$ post.; $33.9 \%$ seg. & cen
\end{tabular}

González et 60 usuarios de 25-55 años de cen- GE1: Terapia cognitivo- conduc- -GE1 y GE2 obtienen mejores resultados que al. (2006) tros de atención primaria tual $(n=$ n.d.) GE3, y éste mejores que GC, en mejora de la sinn.d. GE2: Potenciación de recursos per- tomatología depresiva. n.d. $\quad$ sonales $(n=$ n.d.)

n.d. $\quad$ GE3: Apoyo social $(n=$ n.d. $)$ GC: Lista de espera $(n=$ n.d. $) \quad$ seg. 12 meses.

-Mayores mejorías en sintomatología depresiva en GE que en GC.

-El GE no mejoró en sintomatología depresiva, pero sí depresión, ansiedad y trastornos de adaptación (combinados).

Konnert et 64 residentes en centros de la terce- GE: Programa de Afrontamiento - Diferencias entre GE y GC en post. y seg. a los 3 al. (2009) ra edad del Estrés $(n=35) \quad$ y 6 meses en sintomatología depresiva. $77 \%$ mujeres

Edad media $=81.1(D T=6.8) \quad$ GC: Atención habitual $(n=29)$

-Incidencia: 2 nuevos casos de episodio depresivo mayor GC, frente a 0 de GE, en seguimientos.

Korte et al. 202 adultos mayores procedentes GE: Terapia de revisión de vida $(n$-El GE fue efectivo en reducir la sintomatología (2012) de servicios de salud mental $\quad=100)$ depresiva, en el postratamiento y los seguimientos. GE $80 \%$ mujeres; GC $73.5 \%$ mujeres $\quad$ GC: Atención habitual Edad media: $\mathrm{GE}=63.3(\mathrm{DT}=(n=102)$ -Se encontraron efectos pequeños significativos pa$6.2) ; \mathrm{GC}=63.3(D T=6.8)$ ra síntomas de ansiedad y salud mental positiva. $16.8 \%$

Lara et al. 377 mujeres mexicanas embarazadas GE: Psicoeducativa. Salud Mental -La incidencia acumulativa de depresión a lo largo (2010) 100\% mujeres de Mamás y Bebés $(n=250) \quad$ del seguimiento fue más baja en GE $(10.7 \%)$ que Edad media $=26.9(D T=5.6)$ $63.9 \%$ GC: Atención habitual $(n=127)$ en GC $(25 \%)$.

-Reducción significativa de síntomas depresivos en ambos grupos, pero no efectos significativos del tratamiento.

\begin{tabular}{|c|c|c|c|}
\hline $\begin{array}{l}\text { Le et al. } \\
(2011)\end{array}$ & $\begin{array}{l}217 \text { mujeres embarazadas de alto } \\
\text { riesgo } 18-35 \text { años } \\
100 \% \text { mujeres } \\
\text { Edad media = n.d. } \\
30.9 \%\end{array}$ & $\begin{array}{l}\text { GE: Curso de Mamás y Bebés }(n= \\
\text { 112) } \\
\text { GC: Atención habitual }(n=105)\end{array}$ & $\begin{array}{l}\text {-GE tuvieron significativamente menos síntomas y } \\
\text { menos casos de depresión moderada que GC en } \\
\text { una evaluación preparto, especialmente para las } \\
\text { mujeres que asistieron a todas las sesiones, pero no } \\
\text { en el período posparto. } \\
\text {-La incidencia acumulativa de episodios depresivos } \\
\text { mayores no difirió entre el GE y el GC. }\end{array}$ \\
\hline $\begin{array}{l}\text { Liu et al. } \\
(2009)\end{array}$ & $\begin{array}{l}52 \text { adultos de } 18 \text { a } 58 \text { años } \\
73 \% \text { mujeres } \\
\text { Edad media }=26.4(D T=8.7) \\
22.6 \%\end{array}$ & $\begin{array}{l}\text { GE: Psicoeducativa. Biblioterapia: } \\
\text { Mind Over } \operatorname{Mood}(n=27) \\
\text { GC: Lista de espera }(n=25)\end{array}$ & $\begin{array}{l}\text {-Diferencia en el nivel de depresión conjunto en- } \\
\text { tre los participantes GE-GC (ES }=7.0, d= \\
0.76)^{12} \text {. } \\
\text {-GE efecto fuerte en los síntomas cognitivo- } \\
\text { afectivos de depresión }(\mathrm{ES}=6.6, d=0.82) \text {, pero }\end{array}$ \\
\hline
\end{tabular}

\footnotetext{
${ }^{12}$ Donde ES fue definido como la diferencia entre las medias de las puntuaciones de cambio del pretest al postest en el BDI-II de los grupos experimental y
} control 
no en los somáticos (ES $=0.4, d=0.23$ ).

-Tamaño del efecto de medio a grande para la disponibilidad de recursos aprendidos $(\mathrm{ES}=$-9.9, $d=-0.77)$.

Lynch et al. 29 pacientes de una consulta de me- GE: Terapia de solución de pro- - GE puntuaciones significativamente más bajas en

(1997) dicina familiar blemas telefónica $(n=15)$

$87.5 \%$ mujeres GE; $85.7 \%$ mujeres

GC

GC: No intervención $(n=14)$

Edad media: $\mathrm{GE}=46.8$

$\mathrm{GC}=49.9$

$13.8 \%$

Mossey et al. 89 pacientes de los servicios de sa- GE: Interpersonal Counseling ( $n$

(1996) lud mayores de 60 años =35)

$78.2 \%$ mujeres

Edad media $=71(D T=7.7) \quad$ GC: Atención habitual $(n=41)$

$14.6 \%$

Muñoz et al. 45 mujeres latinas embarazadas de GE: Curso de Mamás y Bebés $(n$

(2007) bajos ingresos =21)

$100 \%$ mujeres

Edad media $=25(D T=4.4) \quad$ GC: Atención habitual + informa-

$9.1 \% \quad$ ción $(n=20)$

Puskar et al. 89 estudiantes de escuelas rurales GE: Terapia cognitivo-conductual -Mejora en la sintomatología depresiva y ciertas

(2003) mayores de 13 años (Teaching Kids to Cope) $(n=$ habilidades de afrontamiento.

$82 \%$ mujeres n.d.)

Edad media $=16(D T=0.9)$

$20.2 \%$

GC: Atención habitual $(n=$ n.d.)

-En GE mayor uso de estrategias de solución de problemas cognitivas

Roberts et al. 189 jóvenes 11-13 años GE: Penn Prevention Program $(n$

(2003) $\quad 50.3 \%$ varones

Edad media $=11.9(D T=0.3)$ $=90)$

$5.3 \%$

GC: Atención habitual $(n=99)$

Roberts et al. 189 jóvenes 11-13 años

(2004)c $\quad 50.3 \%$ varones

Edad media $=11.9(D T=0.3)$ $5.3 \%$

GE: Penn Prevention Program ( $n$ $=90$ )

GC: Atención habitual $(n=99)$

Seligman et 231 estudiantes universitarios de GE: Programa de prevención

al. (1999) primer año

$52 \%$ mujeres

Edad media $=$ n.d.

$10.8 \%$.

cognitivo-conductual $(n=106)$

GC: Sólo evaluación $(n=119)$

-No encontraron diferencias en síntomas depre-

sivos GE y GC en el post. y el seg. 6 meses.

- Diferencias entre GE y GC en ansiedad.

-Diferencias problemas internalizantes y externalizantes en el post., pero no en seg. 6 meses.

en sínto$(60.0 \%$ vs. $35.1 \%)$.

-Efecto positivo del tratamiento también sobre la salud autoinformada, pero no sobre el funciona-

No encontraron diferencias entre GE y GC en sintomatología depresiva o incidencia de depresión mayor.

-No encontraron diferencias significativas GE y GC para sintomatología depresiva en los seguimientos.

-GE tendencia hacia menos episodios depresivos mayores moderados que GC, pero no severos.

-GE menos síntomas depresivos y de ansiedad que GC.

-GE más mejoras en estilo explicativo, desesperanza y actitudes disfuncionales que el GC.

Seligman et 240 estudiantes universitarios de GE: Programa de prevención

$\begin{array}{ll}\text { al. (2007) } & \text { primer año } \\ \text { n.d. } & \text { cognitivo-conductual }(n=113)\end{array}$ n.d.

Edad media $=$ n.d.

GC: Sólo evaluación $(n=127)$

$8.8 \%$

-GE menos sintomatología depresiva que GC pa-

ra BDI en evaluación final y seguimiento a los 6 meses, con tamaños del efecto pequeñosmoderados.

-No encontraron diferencia entre GE y GC en incidencia trastornos depresivos a los 6 meses.

Sheffield et 2584 estudiantes de $9^{\circ}$ curso (de 13 GE1: Universal: Reestructuración al. (2006) a 15 años), 521 en riesgo. cognitiva + entrenamiento solu$54 \%$ mujeres muestra total; $69 \%$ ción de problemas $(n=621)$ (alto mujeres muestra riesgo.

Edad media $=14.3(D T=0.4)$

riesgo $n=126$ )

GE2: Indicada: mismos compo-

$6.2 \%$ muestra total; $5.0 \%$ grupo alto nentes, grupos más pequeños, seriesgo.

siones más largas $(n=636)$ (alto riesgo $n=112$ )

GE3: Intervenciones de

GE1+GE2 de manera sucesiva $(n$

$=722$ ) (alto riesgo $n=134$ )

GC: Sólo evaluación $(n=605)$ (al-

to riesgo $n=149$ )

cias significativas entre los grupos en sintomatología depresiva, ansiedad, problemas externalizantes, habilidades de afrontamiento y ajuste social.

-Todos los estudiantes con síntomas elevados, independientemente de la condición, presentaron disminución de síntomas depresivos con el tiempo, aunque todavía mostraban niveles elevados comparados con la población general a los 12 meses.

GE: Problem Solving for Life $(n$

-GE alto riesgo más decremento en sintomatolo- 


\begin{tabular}{|c|c|c|c|}
\hline$\overline{(2003)}$ & $\begin{array}{l}\text { alto riesgo } \\
\text { GE: } 52.5 \% \text { mujeres; GC: } 50.6 \% \\
\text { mujeres. } \\
\text { Edad media = n.d. } \\
15 \%\end{array}$ & $\begin{array}{l}=751) . \\
\text { GC: Sólo evaluación }(n=749)\end{array}$ & $\begin{array}{l}\text { gía depresiva e incremento en puntuaciones de } \\
\text { solución de problemas que GC }\end{array}$ \\
\hline $\begin{array}{l}\text { Stamp et al. } \\
(1995)\end{array}$ & $\begin{array}{l}144 \text { mujeres embarazadas en riesgo } \\
\text { de depresión postnatal } \\
100 \% \text { mujeres } \\
\text { Edad media: } \mathrm{GE}=25.6(D T=4.4) ; \\
\mathrm{GC}=27.5(D T=5.2) \\
18.1 \%\end{array}$ & $\begin{array}{l}\text { GE: Intervención de apoyo y ex- } \\
\text { presión }(n=73) \\
\text { GC: Atención habitual }(n=71)\end{array}$ & $\begin{array}{l}\text { - No encontraron diferencias GE y GC en } 6 \text { ni } 12 \\
\text { semanas. }\end{array}$ \\
\hline $\begin{array}{l}\text { Stice et al. } \\
(2006)\end{array}$ & $\begin{array}{l}225 \text { adolescentes } \\
70 \% \text { mujeres } \\
\text { Edad media }=18.0 \\
14.7 \%\end{array}$ & $\begin{array}{l}\text { GE1: Prevención cognitivo- } \\
\text { conductual }(n=50) \\
\text { GE2: Intervención grupal de apo- } \\
\text { yo y expresión }(n=19) \\
\text { GE3: Biblioterapia }(n=28) \\
\text { GE4: Escritura expresiva }(n=27) \\
\text { GE5: Diario }(n=34) \\
\text { GC: Lista de espera }(n=67)\end{array}$ & $\begin{array}{l}\text {-GE1, GE2, GE3, GE4 y GE5 mostraron mayo- } \\
\text { res reducciones que GC en la sintomatología de- } \\
\text { presiva en el post. } \\
\text {-Los efectos para GE1 Y GE3 persistieron a los } \\
6 \text { meses. } \\
\text {-GE1. GE2 y GE3 mostraron mayores decre- } \\
\text { mentos en sintomatología depresiva que GE4 y } \\
\text { GE5 en los seguimientos. }\end{array}$ \\
\hline $\begin{array}{l}\text { Stice et al. } \\
(2008)\end{array}$ & $\begin{array}{l}341 \text { jóvenes de } 14-19 \text { años } \\
56 \% \text { mujeres } \\
\text { Edad media }=15.6(D T=1.2) \\
10.0 \%\end{array}$ & $\begin{array}{l}\text { GE1: Prevención cognitivo- } \\
\text { conductual }(n=89) \\
\text { GE2: Intervención grupal de apo- } \\
\text { yo y expresión }(n=88) \\
\text { GE3: Biblioterapia }(n=80) \\
\text { GC: Sólo evaluación }(n=84)\end{array}$ & $\begin{array}{l}\text {-GE1 mejores resultados que GE2, GE3 y GC en } \\
\text { el post., aunque sólo significación estadística a los } \\
6 \text { meses. } \\
\text {-GE2 y GE3 mayores mejorías que GC en sin- } \\
\text { tomatología depresiva en algunos seguimientos. } \\
\text {-GE1, GE2 y GE3 riesgo menor inicio trastorno } \\
\text { depresivo mayor que GC en seg. } 6 \text { meses. } \\
\end{array}$ \\
\hline $\begin{array}{l}\text { Stice et al. } \\
(2010)^{\mathrm{d}}\end{array}$ & $\begin{array}{l}341 \text { jóvenes de } 14-19 \text { años } \\
56 \% \text { mujeres } \\
\text { Edad media }=15.6(D T=1.2) \\
22.3 \%\end{array}$ & $\begin{array}{l}\text { GE1: Prevención cognitivo- } \\
\text { conductual }(n=89) \\
\text { GE2: Intervención grupal de apo- } \\
\text { yo y expresión }(n=88) \\
\text { GE3: Biblioterapia }(n=80)\end{array}$ & $\begin{array}{l}\text {-GE1 mayores reducciones en sintomatología } \\
\text { depresiva que GC en seg. } 1 \text { año y que GE3 en } \\
\text { seg. } 1 \text { año y } 2 \text { años. } \\
\text {-GE2 mayores reducciones que GE3 en seg. } 2 \\
\text { años. } \\
\text {-Riesgo para el inicio de depresión en seg. } 2 \text { años } \\
\text { más bajo para GE1 y GE3 que GC. }\end{array}$ \\
\hline $\begin{array}{l}\text { Van } \begin{array}{l}\text { Voor- } \\
\text { hees }\end{array} \text { et al. } \\
(2008)\end{array}$ & $\begin{array}{l}84 \text { jóvenes de } 14-21 \text { años } \\
\text { Aprox. } 50 \% \text { de cada sexo. } \\
\text { Edad media: GE1 = } 17.4(D T= \\
2.2) ; \text { GE2 }=17.3(D T=2.0) \\
4.8 \%\end{array}$ & $\begin{array}{l}\text { GE1: Entrevista motivacional con } \\
\text { médico de atención primaria }+ \\
\text { programa internet }(n=40) \\
\begin{array}{l}\text { GE2: Consejo breve }+ \text { programa } \\
\text { de internet }(n=43)\end{array}\end{array}$ & $\begin{array}{l}\text {-GE1 y GE2 mostraron disminuciones en sintoma- } \\
\text { tología depresiva en el post. con respecto al pretra- } \\
\text { tamiento. }\end{array}$ \\
\hline $\begin{array}{l}\text { Van Voor- } \\
\text { hees et al. } \\
(2009)^{\mathrm{e}}\end{array}$ & $\begin{array}{l}84 \text { jóvenes de } 14-21 \text { años } \\
\text { Aproximadamente } 50 \% \text { de cada } \\
\text { sexo } \\
\text { Edad media: } \mathrm{GE} 1=17.4(D T= \\
2.2) ; \mathrm{GE} 2=17.3(D T=2.0) \\
4.8 \%\end{array}$ & $\begin{array}{l}\text { GE1: Entrevista motivacional con } \\
\text { médico de atención primaria }+ \\
\text { programa internet }(n=40) \\
\text { GE2: Consejo breve + programa } \\
\text { de internet }(n=43)\end{array}$ & $\begin{array}{l}\text {-GE1 y GE2 mostraron disminuciones en sinto- } \\
\text { matología depresiva en el seguimiento a las } 12 \\
\text { semanas. } \\
\text {-GE1 disminución en pensamientos autolesivos y } \\
\text { desesperanza, y menos probabilidad de experi- } \\
\text { mentar un episodio depresivo que GE2. }\end{array}$ \\
\hline $\begin{array}{l}\text { Van't Veer- } \\
\text { Tazelaar et al. } \\
(2009)\end{array}$ & $\begin{array}{l}170 \text { personas mayores a partir de } \\
75 \text { años } \\
73.5 \% \text { mujeres. } \\
\text { Edad media: } \mathrm{GE}=81.8(D T= \\
3.8) ; \mathrm{GC}=81.1(D T=3.5) \\
17.6 \%\end{array}$ & $\begin{array}{l}\text { GE: Curso de Afrontamiento de } \\
\text { la Depresión y la Ansiedad }(n= \\
\text { 86) } \\
\text { GC: Atención habitual }(n=84)\end{array}$ & $\begin{array}{l}\text {-GE redujo a la mitad la incidencia de trastornos } \\
\text { depresivos y de ansiedad. }\end{array}$ \\
\hline $\begin{array}{l}\text { Van't Veer- } \\
\text { Tazelaar et al. } \\
(2011)^{\mathrm{f}}\end{array}$ & $\begin{array}{l}170 \text { personas mayores a partir de } \\
75 \text { años } \\
73.5 \% \text { mujeres. } \\
\text { Edad media: } \mathrm{GE}=81.8(D T= \\
3.8) ; \mathrm{GC}=81.1(D T=3.5) \\
17.6 \%\end{array}$ & $\begin{array}{l}\text { GE: Curso de Afrontamiento de } \\
\text { la Depresión y la Ansiedad }(n= \\
\text { 86) } \\
\text { GC: Atención habitual }(n=84)\end{array}$ & $\begin{array}{l}\text {-La incidencia acumulativa de trastornos depresivos } \\
\text { mayores o ansiedad a los } 24 \text { meses se redujo a la } \\
\text { mitad por la intervención. } \\
\text { - Número necesario de sujetos a tratar (NNT) fue } \\
\text { de } 5 \text {. }\end{array}$ \\
\hline $\begin{array}{l}\text { Vázquez et } \\
\text { al. (2013) }\end{array}$ & $\begin{array}{l}173 \text { cuidadoras no profesionales } \\
100 \% \text { mujeres } \\
\text { Edad media: } \mathrm{GE}=53.6(D T= \\
10.1) ; \mathrm{GC}=54.3(D T=8.2) \\
3.4 \% \mathrm{GE}, 1.2 \% \mathrm{GC}\end{array}$ & $\begin{array}{l}\text { GE: Solución de problemas } \\
(n=89) \\
\text { GC: Atención habitual } \\
(n=84)\end{array}$ & $\begin{array}{l}\text { - En el postratamiento hubo una mayor reducción } \\
\text { en la incidencia de depresión, los síntomas depresi- } \\
\text { vos, el malestar emocional y la sobrecarga del cui- } \\
\text { dador, así como una mayor recuperación con signi- } \\
\text { ficación clínica, de las participantes del grupo de in- }\end{array}$ \\
\hline
\end{tabular}




\begin{tabular}{|c|c|c|c|}
\hline \multirow{2}{*}{$\begin{array}{l}\text { Vázquez } \\
\text { al. (2012) }\end{array}$} & \multirow[b]{2}{*}{$\begin{array}{l}133 \text { estudiantes universitarios } \\
82.0 \% \text { mujeres } \\
\text { Edad media: } \text { GE1 }=23.9(D T= \\
\text { 5.4); GE2 }=22.5(D T=3.1) \\
\text { 7.1\% GE1, } 6.3 \% \text { GE2 }\end{array}$} & & \multirow[b]{2}{*}{$\begin{array}{l}\text { tervención respecto al control. } \\
\text {-No hubo diferencias entre las dos condiciones de } \\
\text { intervención en el seguimiento a los } 6 \text { meses, pero } \\
\text { sí hubo diferencias con respecto a la línea base en } \\
\text { sintomatología depresiva y de ansiedad, y una baja } \\
\text { incidencia de episodios depresivos. }\end{array}$} \\
\hline & & $\begin{array}{l}\text { GE1: Adaptación del Curso de } \\
\text { Prevención de la Depresión }(n= \\
\text { 70) } \\
\text { GE2: Entrenamiento en Relaja- } \\
\text { ción }(n=63)\end{array}$ & \\
\hline $\begin{array}{l}\text { Westerhof et } \\
\text { al. (2010) }\end{array}$ & $\begin{array}{l}171 \text { participantes de } 50 \text { años o } \\
\text { más } \\
73.5 \% \text { mujeres GE; } 71.6 \% \text { muje- } \\
\text { res GC } \\
\text { Edad media: GE }=64.4(D T= \\
6.7) ; \mathrm{GC}=64.2(D T=8.1) \\
18.1 \%\end{array}$ & $\begin{array}{l}\text { GE: Terapia de revisión de vida } \\
(n=83) \\
\text { GC: Vídeo sobre hacerse mayor }(n \\
=88)\end{array}$ & $\begin{array}{l}\text {-GE mejoró más en significado personal que GC } \\
\text { en postratamiento, pero no en seg., mediatizan- } \\
\text { do los efectos del programa sobre la sintomato- } \\
\text { logía depresiva. }\end{array}$ \\
\hline $\begin{array}{l}\text { Willemse et } \\
\text { al. (2004) }\end{array}$ & $\begin{array}{l}216 \text { pacientes de atención prima- } \\
\text { ria } 18-65 \text { años. } \\
\text { n.d. } \\
\text { Edad media = n.d. } \\
25.5 \%\end{array}$ & $\begin{array}{l}\text { GE: Curso de Afrontamiento de } \\
\text { la Depresión telefónico }(n=107) \text {. } \\
\text { GC: Atención habitual }(n=109) \text {. }\end{array}$ & $\begin{array}{l}\text {-En seg. } 12 \text { meses, la incidencia de trastorno de- } \\
\text { presivo mayor fue más baja en GE }(12 \%) \text { que en } \\
\text { GC }(18 \%) \text {. } \\
\text {-También se encontraron efectos significativos } \\
\text { pero pequeños en sintomatología depresiva y as- } \\
\text { pectos en calidad de vida relacionada con salud. }\end{array}$ \\
\hline $\begin{array}{l}\text { Young et al. } \\
(2006)\end{array}$ & $\begin{array}{l}41 \text { adolescentes de } 7^{\circ} \text { a } 10^{\circ} \text { curso } \\
85.4 \% \text { mujeres } \\
\text { Edad media }=13.4(D T=1.2) \\
2.4 \%\end{array}$ & $\begin{array}{l}\text { GE1: Psicoterapia interpersonal. } \\
\text { Adolescent Skills Training (Teen } \\
\text { Talk) }(n=27) \\
\text { GE2: Consejo escolar }(n=14)\end{array}$ & $\begin{array}{l}\text {-GE1 tuvieron menos síntomas depresivos y un } \\
\text { mejor funcionamiento conjunto post. y en el seg., } \\
\text { y una tendencia hacia menor incidencia de tras- } \\
\text { tornos depresivos que GE2. }\end{array}$ \\
\hline $\begin{array}{l}\text { Young et al. } \\
(2010)\end{array}$ & $\begin{array}{l}57 \text { estudiantes de } 9^{\circ} \text { y } 10^{\circ} \text { grado, de } \\
13-17 \text { años } \\
59.7 \% \text { mujeres. } \\
\text { Edad media }=14.5(D T=0.8) \\
24.6 \%\end{array}$ & $\begin{array}{l}\text { GE1: Psicoterapia interpersonal. } \\
\text { Adolescent Skills Training (Teen } \\
\text { Talk) }(n=36) \\
\text { GE2: Consejo escolar-atención ha- } \\
\text { bitual }(n=21)\end{array}$ & $\begin{array}{l}\text {-GE1 mayores reducciones en la sintomatología } \\
\text { depresiva y funcionamiento conjunto que GE2 } \\
\text { en post. } \\
\text {-Durante el seg., las tasas de cambio se redujeron } \\
\text { para GE1, pero se incrementaron para GE2. } \\
\text {-En el seguimiento a los } 12 \text { meses no hubo dife- } \\
\text { rencias entre GE1 y GE2. } \\
\text {-GE1 menos incidencia depresión en seg. } 6 \text { meses. }\end{array}$ \\
\hline $\begin{array}{l}\text { Zlotnick et } \\
\text { al. (2001) }\end{array}$ & $\begin{array}{l}37 \text { mujeres embarazadas } \\
100 \% \text { mujeres } \\
\text { Edad media }=23.4(D T=4.4) \\
5.4 \%\end{array}$ & $\begin{array}{l}\text { GE: Psicoterapia interpersonal. } \\
\text { Survival Skills for New Moms }(n \\
=17) \text {. } \\
\text { GC: Atención habitual }(n=18) \text {. }\end{array}$ & $\begin{array}{l}-3 \text { meses después del parto, } 33 \% \text { del GC había } \\
\text { desarrollado depresión mayor, frente a } 0 \% \text { del GE. }\end{array}$ \\
\hline
\end{tabular}

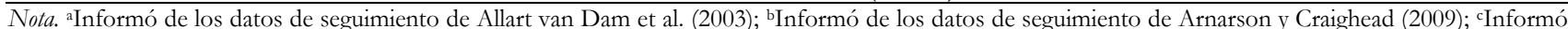
de los datos de seguimiento de Roberts et al. (2003); dInformó de los datos de seguimiento de Stice et al. (2008); eInformó de los datos de seguimiento de Van Voorhees et al. (2008); Informó de los datos de seguimiento de Van't Veer-Tazelaar et al. (2009); GE = Grupo experimental; GC = grupo control; Post. = Postratamiento; Seg. = Seguimiento; n.d. = no hay datos.

\section{Análisis Metodológico de los Estudios}

En relación con la metodología empleada en los ensayos analizados, el tamaño muestral de los estudios varió de 29 (Lynch et al., 1997) a 2584 personas (Sheffield et al., 2006). Este último incluyó población sin riesgo y con riesgo.

En la mayoría de los estudios no se informó del ocultamiento de la asignación. Sin embargo, se explicitó en doce estudios (Clarke et al., 2001; Dozeman et al., 2012; Fledderus et al., 2012; Korte et al., 2012; Lara et al., 2010; Le et al., 2011; Lynch et al., 1997; Muñoz et al., 2007; Stamp et al., 1995; Van Voorhees et al., 2008, 2009; Vázquez et al., 2012, 2013) y no se realizó en un estudio (Seligman et al., 1999). En 15 estudios se realizó el ciego de los evaluadores (Dozeman et al., 2012; Garber et al., 2009; Muñoz et al., 2007; Roberts et al., 2003, 2004; Seligman et al., 1999, 2007; Stamp et al., 1995; Stice et al., 2008, 2010; Van Voorhees et al., 2008, 2009; Van't Veer-Tazelaar, 2009, 2011;Vázquez et al., 2012,
2013; Willemse et al., 2004; Young et al., 2006, 2010), no se realizó en dos estudios (Clarke et al., 2001; Konnert et al., 2009) y no se informó en el resto de los trabajos. No se realizó el ciego de los sujetos en 12 estudios (Clarke et al., 1995; Clarke et al., 2001; Dozeman et al., 2012; Garber et al., 2009; Seligman, 1999, 2007; Stamp et al., 1995; Van Voorhees et al., 2008, 2009; Willemse et al., 2004; Young et al., 2006, 2010; Zlotnick et al., 2001), se realizó en un estudio (Muñoz et al., 2007) y quedó poco claro o no se informó en el resto.

La mayoría de los estudios presentaron criterios de inclusión claros. Los criterios de inclusión consistieron en la presencia de sintomatología depresiva elevada en función de la puntuación en instrumentos validados para su evaluación, pero sin reunir los criterios para un trastorno depresivo mayor, en 26 estudios (con sus correspondientes seguimientos) (Allart-van Dam et al., 2003, 2007; Clarke et al., 1995, 2001; Dobson et al., 2010; Dozeman et al., 2012; Gillham et al., 
2006; González et al., 2006; Konnert et al., 2009; Liu et al., 2009; Lynch et al., 1997; Mossey et al., 1996; Roberts et al., 2003, 2004; Seligman et al., 2007; Sheffield et al., 2006; Spence et al., 2003; Stamp et al., 1995; Stice et al., 2006, 2008, 2010; Van't Veer-Tazelaar, 2009, 2011; Vázquez et al., 2012, 2013; Westerhof et al., 2010; Willemse et al., 2004; Young et al., 2006, 2010; Zlotnick et al., 2001); o bien presentar síntomas subumbral de depresión definidos como presencia de síntomas nucleares al menos unos días en las dos últimas semanas, sin reunir los criterios para un trastorno depresivo mayor (Van Voorhees et al., 2008, 2009). Otros estudios incluyeron criterios adicionales: presencia de trastorno depresivo mayor pasado en un estudio (Muñoz et al., 2007); presencia de estilo atribucional pesimista en un estudio (Arnarson y Craighead, 2009, 2011); presencia de episodios depresivos mayores paternos, o un episodio depresivo mayor en remisión durante dos meses en la persona participante en el programa, en un estudio (Garber et al., 2009); tener más de 18 años y síntomas de ansiedad de leves a moderados en un estudio (Fledderus et al., 2012); ser mayor de 55 años en un estudio (Korte et al., 2012); y no estar atravesando una situación de duelo en un estudio (Puskar et al., 2003). Dos estudios señalaron como criterio de inclusión estar en estado de gestación (Lara et al., 2010; Stamp et al., 1995). Dos de estos estudios (Le et al., 2011; Seligman et al., 1999) combinaron varios de estos criterios de inclusión.

El cumplimiento del programa de prevención se mantuvo con integridad comprobada en 15 estudios (Allart-van Dam et al., 2003, 2007; Clarke et al., 1995, 2001; Dobson et al., 2010; Garber et al., 2009; Gillham et al., 2006; Korte et al., 2012; Liu et al., 2009; Puskar et al., 2003; Roberts et al., 2003, 2004; Seligman et al., 1999; Spence et al., 2003; Stice et al., 2008, 2010; Van Voorhees et al., 2008, 2009; Vázquez et al., 2012); se informó parcialmente en un estudio (Sheffield et al., 2006); se informó su toma en consideración, pero sin especificar los datos de adherencia al tratamiento por parte de los terapeutas en cuatro estudios (Konnert et al., 2009; Lara et al., 2010; Le et al., 2011; Seligman et al., 2007) y no se informó en el resto de los estudios.

Los períodos de seguimiento variaron de cuatro publicaciones que informaron de los resultados en la evaluación postratamiento (Allart-van Dam et al., 2003; Lynch et al., 1997; Van Voorhees et al., 2008; Vázquez et al., 2013), a aquellos que informaron de resultados hasta los 9 meses (Arnarson y Craighead, 2009; Dobson et al., 2010; Fledderus et al., 2012; Garber et al., 2009; Konnert et al., 2009; Korte et al., 2012; Lara et al., 2010; Liu et al., 2009; Roberts et al., 2003; Stamp et al., 1995; Stice et al., 2006, 2008; Van Voorhees et al., 2009; Van't Veer-Tazelaar et al., 2011; Vázquez et al., 2012; Westerhof et al., 2010; Young et al., 2006; Zlotnick et al., 2001) y aquellos que informaron de resultados de 10 a 36 meses (Allart-van Dam et al., 2007; Arnarson y Craighead, 2011; Clarke et al., 1995, 2001; Dozeman et al., 2012; Gillham et al., 2006; González et al., 2006; Le et al., 2011; Mossey et al., 1996; Muñoz et al., 2007; Puskar et al., 2003; Roberts et al., 2004; Seligman et al., 1999, 2007; Shef- field et al., 2006; Spence et al., 2003; Stice et al., 2010; Van't Veer-Tazelaar et al., 2009; Willemse et al., 2004; Young et al., 2010).

En 24 estudios se informó de que se habían evaluado los diagnósticos de trastorno depresivo mayor (Allart-van Dam et al., 2003, 2007; Arnarson y Craighead, 2009, 2011; Clarke et al., 1995, 2001; Dozeman et al., 2012; Garber et al., 2009; Gillham et al., 2006; Konnert et al., 2009; Korte et al., 2012; Lara et al., 2010; Le et al., 2011; Muñoz et al., 2007; Puskar et al., 2003; Seligman et al., 1999, 2007; Spence et al., 2003; Stice et al., 2008, 2010; Willemse et al., 2004; Young et al., 2006, 2010; Van Voorhees et al., 2008, 2009; Vázquez et al., 2012, 2013; Zlotnick et al., 2001), y en dos se informó de diagnósticos de trastornos depresivos mayor y menor probables en función del punto de corte en una escala, pero sin emplear criterios diagnósticos estandarizados (Stamp et al., 1995; Stice et al, 2006). En el resto de los ensayos no se analizó la incidencia de trastornos depresivos.

Se realizó un análisis del tipo intención de tratar en 22 estudios (Allart-van Dam et al., 2003, 2007; Clarke et al., 1995, 2001; Dobson et al., 2010; Dozeman et al., 2012; Fledderus et al., 2012; Garber et al., 2009; Gillham et al., 2006; Korte et al., 2012; Lara et al., 2010; Le et al., 2011; Liu et al., 2009; Mossey et al., 1996; Stamp et al., 1995; Stice et al., 2008, 2010; Van Voorhees et al., 2008, 2009; Van't VeerTazelaar et al., 2009, 2011; Vázquez et al., 2012, 2013; Willemse et al., 2004; Young et al., 2006, 2010), no se realizó en dos estudios (Konnert et al., 2009; Lynch et al., 1997) y quedó poco claro o no se informó en el resto.

Los estudios revisados informaron mayoritariamente de cifras de abandono entre un 20\% y un 30\% (Allart-van Dam et al., 2003, 2007; Arnarson y Craighead, 2009; Clarke et al., 1995; Gillham et al., 2006; Liu et al., 2009), o incluso superiores en seguimientos más largos. Clarke et al. (2001) y Gillham et al. (2006) informaron de un abandono superior al $33 \%$ en el seguimiento a los 24 meses. No obstante, algunos estudios mostraron cifras de abandono mucho más reducidas. Por ejemplo, Garber et al. (2009) encontraron un abandono del $4.8 \%$ en el postratamiento y el $9.5 \%$ en el seguimiento a los 6 meses. Roberts et al. $(2003,2004)$ y Young et al. (2006), con población escolar obtuvieron cifras de entre un $2.4 \%$ y un $5.3 \%$ en seguimientos de hasta 30 meses, y Muñoz et al. (2007) y Vázquez et al. (2012) porcentajes de abandono entre el $6.3 \%$ y el $9.1 \%$ en el seguimiento a los 6 meses. En los estudios de Seligman et al. (1999, 2007) las pérdidas de participantes fueron también reducidas (de un $10.8 \%$ y un $8.8 \%$, respectivamente), pero se incentivó su participación.

\section{Análisis de los Resultados de los Estudios}

Sintomatología depresiva y episodios depresivos

Para aquellos que informaron del tamaño del efecto para la sintomatología y trastornos depresivos, evaluado a través 
de la $d$ de Cohen ${ }^{11}$, los programas obtuvieron tamaños del efecto diversos: siete estudios encontraron tamaños del efecto pequeños (Clarke et al., 1995; Le et al., 2011; Muñoz et al., 2007; Seligman et al., 1999, 2007; Stice et al., 2008, 2010; Willemse et al., 2004); cinco encontraron tamaños del efecto moderados (Allart-van Dam et al., 2003; Korte et al., 2012; Liu et al., 2009; Van Voorhees et al., 2008; Young et al., 2010); dos estudios encontraron tamaños del efecto moderados-grandes en función de los distintos seguimientos y grupos (Fledderus et al., 2012; Vázquez et al., 2012); y tres estudios encontraron un tamaño medio del efecto grande (Konnert et al., 2009; Vázquez et al., 2013; Young et al., 2006).

En cuanto a la incidencia de trastornos depresivos, en el postratamiento, tres estudios (Korte et al., 2012; Le et al., 2011; Van Voorhees et al., 2008) no encontraron diferencias significativas en la incidencia de trastornos depresivos entre los grupos de intervención y los de comparación, mientras otro sí encontró diferencias (Vázquez et al., 2013). En el seguimiento de 3 a 6 meses, seis estudios de una intervención específica (Clarke et al., 1995; Puskar et al., 2003; Seligman et al., 2007; Spence et al., 2003; Stice et al., 2006, 2008) no demostraron un efecto significativo sobre la aparición de nuevos episodios de depresión, mientras otros cinco encontraron una menor incidencia de depresión en el grupo experimental, frente a un grupo de atención habitual (Arnarson y Craighead, 2009; Garber et al., 2009; Lara et al., 2010; Young et al., 2010; Zlotnick et al., 2001). En seguimientos de los 10 a los 12 meses, cinco estudios de intervenciones específicas (Arnarson y Craighead, 2011; Clarke et al., 1995, 2001; Dozeman et al., 2012; Willemse et al., 2004) informaron una reducción significativa de la aparición de nuevos episodios de depresión en los grupos de intervención frente a los control, mientras que uno no encontró diferencias significativas entre los dos grupos (Allart-van Dam et al., 2007). Un estudio a los 24 meses (Stice et al., 2010) encontró diferencias significativas, mientras que un estudio a los 18 meses (Young et al., 2010), tres estudios a los 24 meses (Clarke et al., 2001; Gillham et al., 2006; Van't Veer-Tazelaar et al., 2011) y un estudio a los 36 meses (Seligman et al., 1999) no demostraron una diferencia significativa. Tres estudios (Konnert et al., 2009; Muñoz et al., 2007; Young et al., 2006) encontraron una tendencia hacia un menor número de diagnósticos de trastornos depresivos en el grupo experimental, pero este resultado se vio limitado por el pequeño tamaño muestral. Un estudio (Stamp et al., 1995) informó de la inexistencia de diferencias significativas en los diagnósticos probables de trastornos depresivos mayor y menor a las 6 semanas, encontrándose diferencias en cambio a las 12 semanas y a los 6 meses; existen limitaciones metodológicas que recomiendan considerar estos datos con cautela. Un estudio (Van Voorhees et al., 2009) encontró una reducción de la depresión

\footnotetext{
11 Se trata de un indicador de la magnitud de la diferencia entre dos medias. Cohen (1988) propone los siguientes valores como orientativos: $d=0.20$ (efecto pequeño); $d=0.50$ (efecto moderado); $d=0.80$ (efecto grande).
}

clínicamente significativa a las 2 semanas en los dos grupos de participantes en el estudio. Un estudio (Vázquez et al., 2012) encontró una incidencia baja de depresión mayor en el postratamiento y el seguimiento a los 6 meses en los dos grupos de intervención.

Por lo que respecta a los estudios que informaron de la sintomatología depresiva, siete estudios mostraron beneficios significativos del programa en el postratamiento (Clarke et al., 1995; Liu et al., 2009; Spence et al., 2003; Stice et al., 2006; Vázquez et al., 2013; Young et al., 2006, 2010), con respecto a grupos de atención habitual o lista de espera; mientras que cuatro no los mostraron (Le et al., 2011; Lynch et al., 1997; Roberts et al., 2003; Stice et al., 2008). Van Voorhees et al. (2008) y Dobson et al. (2010) encontraron diferencias significativas para los grupos de intervención en el tiempo, pero no entre los dos grupos de intervención. Once estudios se mostraron eficaces en la reducción de la sintomatología en seguimientos cortos hasta los 9 meses (Allart-van Dam et al., 2003; Garber et al., 2009; Fledderus et al., 2012; González et al., 2006; Korte et al., 2012; Mossey et al., 1996; Puskar et al., 2003; Seligman et al., 2007; Stice et al., 2008; Young et al., 2006; Zlotnick et al., 2001) con respecto a grupos control de atención habitual, lista de espera, o no intervención, mientras que seis estudios (Gillham et al., 2006; Lara et al., 2010; Muñoz et al., 2007; Roberts et al., 2003; Sheffield et al., 2006; Stamp et al., 1995), no encontraron reducciones significativas frente a grupos control de intervención mínima, atención habitual o no intervención; un estudio (Konnert et al., 2009), mostró resultados poco claros; Vázquez et al. (2012) encontraron diferencias intragrupo en el seguimiento a los 6 meses respecto al pretratamiento en ambos grupos, pero no entre las dos condiciones activas; Van Voorhees et al. (2009) encontraron diferencias en el seguimiento a largo plazo con respecto al pretratamiento, pero no entre los dos grupos de intervención. En seguimientos de 10 a 36 meses, siete ensayos mostraron eficacia en la reducción de la sintomatología depresiva con respecto a un grupo control de atención habitual (Allart- van Dam et al.,2007; Arnarson y Craighead, 2011; González et al., 2006; Puskar et al., 2003; Seligman et al., 1999; Stice et al., 2010; Willemse et al., 2004), mientras que otros siete estudios (Clarke et al., 2001; Gillham et al., 2006; Mossey et al., 1996; Muñoz et al., 2007; Roberts et al., 2004; Sheffield et al., 2006; Young et al., 2010) no encontraron diferencias significativas entre el grupo experimental y el grupo control.

\section{Otros resultados}

Diez estudios informaron del efecto que tuvo el programa de intervención sobre los síntomas de ansiedad (Dobson et al., 2010; Dozeman et al., 2012; Fledderus et al., 2012; Gillham et al., 2006; Korte et al., 2012; Lara et al., 2010; Seligman et al., 1999, 2007; Van't Veer-Tazelaar et al., 2011; Vázquez et al., 2012). Se utilizaron diversas medidas y todos ellos informaron de una reducción significativa de los síntomas; encontrándose en los estudios de Gillham et al. (2006) 
y Sheffield et al. (2006), que incluyeron grupos con distintos niveles de sintomatología, para el grupo de alto riesgo. Sin embargo, Dozeman et al. (2012) no encontraron un efecto significativo de la intervención sobre la incidencia de trastornos de ansiedad.

Se informó una serie de otros resultados, la mayoría de los cuales fueron variables cognitivas. Dos estudios informaron de un efecto de las intervenciones psicológicas específicas y generales en comparación con la ausencia de intervención, en relación con el estilo atribucional (Seligman et al, 1999, 2007). Un estudio informó de mejorías en el estilo explicativo para los acontecimientos negativos, pero sólo en mujeres (Gillham et al., 2006). Dos de los estudios informaron de que la intervención produjo menos pensamientos negativos en comparación con la atención habitual (Allart-van Dam et al., 2003, 2007; Sheffield et al., 2006), o bien menos actitudes disfuncionales en el postratamiento y a los 6 meses, pero no a los 3 meses (Konnert et al., 2009). Dos estudios informaron de una disminución de la desesperanza en relación con la ausencia de intervención (Seligman et al., 1999), o en relación con un grupo de tratamiento alternativo (Van Voorhees et al., 2008, 2009). Tres estudios informaron de que la autoestima había mejorado significativamente en relación con un grupo de atención habitual (Allart-van Dam et al., 2003, 2007; Lynch et al., 1997), o con respecto al pretratamiento (Dobson et al., 2010) y otro no encontró efectos significativos (Seligman et al., 1999). Un estudio (Liu et al., 2009) encontró una mejoría significativa en la disponibilidad de recursos aprendidos, y una mejoría más importante para la dimensión cognitivo-afectiva de la depresión, frente a la somática. Un estudio encontró efectos significativos sobre el nivel de actividades agradables en comparación con un grupo de atención habitual (Allart-van Dam et al., 2003, 2007). Cuatro estudios encontraron que el grupo experimental había mejorado significativamente su salud mental general, salud mental positiva o salud autoinformada en comparación con un grupo de atención habitual (Allart-van Dam et al., 2003; Korte et al., 2012; Mossey et al., 1996; Vázquez et al., 2013), en distintos momentos de evaluación. Un estudio encontró un mejor funcionamiento conjunto en el postratamiento y en el seguimiento a los 6 meses, que no se mantuvo en el seguimiento a los 12 y 18 meses (Young et al., 2010). Un estudio (Fledderus et al., 2012) encontró reducciones significativas en la fatiga y la evitación experiencial, y mejorías en la salud mental positiva y conciencia plena.

Un estudio encontró efectos significativos del programa sobre las habilidades sociales, frente a un grupo de atención habitual (Allart-van Dam et al., 2003, 2007), y un estudio encontró una reducción en los pensamientos autolesivos (Van Voorhees et al., 2009). Dos estudios encontraron efectos significativos sobre la frecuencia de apoyo social (Allart-van Dam et al., 2003, 2007) y el ajuste social a los 6 meses (Stice et al., 2008), y un estudio sobre el abuso de sustancias (Stice et al., 2008). Un estudio encontró resultados significativos en la adquisición de significado personal (Westerhof et al., 2010) como variable mediadora de los efectos sobre la sin- tomatología depresiva. Otros estudios no encontraron efectos del programa sobre el apoyo social percibido (Allart-van Dam et al., 2003), el funcionamiento físico y social (Mossey et al., 1996), el ajuste social en los seguimientos a 1 y 2 años (Stice et al., 2010), sobre trastornos no afectivos (Clarke et al., 1995), o en el uso de los servicios de salud mental (Garber et al., 2009). Un estudio (Vázquez et al., 2013) mostró una reducción significativa de la sobrecarga del cuidador en el grupo de intervención.

\section{Discusión}

El objetivo de esta revisión fue analizar la evidencia de la eficacia de las intervenciones de prevención indicada en la reducción de la sintomatología depresiva y la incidencia de depresión. Los estudios analizados en el presente trabajo se dirigieron a participantes de 11 a 91 años de edad y un $47.2 \%$ procedentes de EEUU; evaluaron fundamentalmente intervenciones psicológicas de orientación cognitivoconductual (83.3\% de los estudios), con un promedio de 910 sesiones y una duración media por sesión de 90 minutos, que fueron aplicados por profesionales cualificados de distintas áreas (47.2\% por psicólogos o estudiantes de doctorado de psicología).

Del total de estudios incluidos en la revisión, aproximadamente el $70 \%$ hallaron efectos positivos de las intervenciones de prevención indicada sobre la sintomatología depresiva en el postratamiento. En general esos resultados se pueden considerar como moderadamente satisfactorios, puesto que, aunque informan de efectos positivos de las intervenciones frente a grupos control lista de espera o atención habitual en sintomatología depresiva, la mayoría alcanzaron tamaños del efecto pequeños (menores de 0.50), como ocurrió en los estudios de prevención indicada del metaanálisis Jané-Llopis, Hosman, Jenkins y Anderson (2003). En torno al $60 \%$ de esos estudios encontraron resultados positivos en la disminución de la sintomatología depresiva en los seguimientos a largo plazo. En un estudio (Seligman et al., 1999) los efectos preventivos se mantenían en un período de seguimiento a los 3 años. Estos datos apoyan la observación realizada por Horowitz y Garber (2006) en cuanto a que la erosión de los efectos de este tipo de programas, de producirse, lo hace poco después del final de la intervención.

Respecto a los efectos de las intervenciones sobre la incidencia de la depresión, 24 trabajos y sus correspondientes seguimientos proporcionaron datos sobre la incidencia (más dos trabajos más sobre depresión probable), encontrando resultados contradictorios. Así, por ejemplo, con una versión del programa CWD, Clarke et al. (1995) encontraron una incidencia acumulativa de $14.5 \%$ para el grupo que recibió el programa de prevención y un $25.7 \%$ para el grupo control de atención habitual. Vázquez et al. (2013), empleando solución de problemas, encontraron una incidencia de $4.5 \%$ para el grupo experimental en el postratamiento. Algunos otros programas de prevención indicada informaron de resultados similares en la reducción de la incidencia de la depresión 
comparados con atención habitual (Clarke et al., 2001; Garber et al., 2009; Willemse et al., 2004). Sin embargo, la mayoría de los estudios que analizaron la variable incidencia no encontraron un efecto significativo de las intervenciones en la aparición de nuevos episodios de depresión.

La mayoría de los estudios no aportaron datos acerca de la significación clínica de las intervenciones. No obstante, en aquellos estudios que lo hicieron, los resultados fueron alentadores. Así, Liu et al. (2009) encontraron un porcentaje de sujetos clínicamente recuperados del $48 \%$ en el postratamiento para el grupo de tratamiento. En Fledderus et al. (2012) el porcentaje de sujetos con cambio clínicamente recuperados fue de $34 \%$ y $39 \%$ para los grupos activos en el postratamiento, cifras que alcanzaron un $80.9 \%$ en Vázquez et al. (2013) para el grupo de solución de problemas en este momento de evaluación. Korte et al. (2012) encontraron una recuperación con significación clínica del $45.6 \%$ en el postratamiento, y el $51.5 \%$ en el seguimiento a los 3 meses. Vázquez et al. (2012), comparando dos grupos activos, encontraron cifras entre 39.7 y $62.9 \%$ en el postratamiento, 65.1 y $54.3 \%$ en el seguimiento a los 3 meses, y $47.6 \%$ y $45.7 \%$ en el seguimiento a los 6 meses. Stice et al. (2008) obtuvieron un $43 \%$ de sujetos clínicamente recuperados para el grupo de terapia cognitivo-conductual en el seguimiento a los 6 meses. En Konnert et al. (2009) y Mossey et al. (1996) se informó de una significación clínica de $95 \%$ y $60.6 \%$ en el seguimiento a los 6 meses, respectivamente. Por el contrario, los grupos de comparación en los que no se utilizó ningún tratamiento activo (lista de espera, no tratamiento o atención habitual), los porcentajes de sujetos clínicamente recuperados oscilaron entre el $6 \%$ para los grupos de no tratamiento (Fledderus et al., 2012) y el 35.1\% para grupos de atención habitual (Mossey et al., 1996).

Algunos trabajos informaron también de consecuencias sobre otras variables clínicas. Especialmente interesantes fueron los resultados obtenidos en relación a la reducción de la ansiedad. Todos los estudios que informaron de los efectos de sus intervenciones sobre la sintomatología de ansiedad (p.e., Dobson et al., 2010; Gillham et al., 2006; Lara, et al., 2010; Seligman et al., 1999, 2007; Sheffield et al., 2006; Vázquez et al., 2012), obtuvieron resultados positivos, lo que aporta evidencia sólida acerca de la utilidad de los programas de prevención indicada no sólo en la prevención de la depresión, sino también de la sintomatología de ansiedad. Una posible explicación a este hallazgo es la elevada comorbilidad entre sintomatología depresiva y de ansiedad subclínicas; es más, existe evidencia de que la presencia de ansiedad inicial elevada podría ser un factor de riesgo para el desarrollo de un trastorno depresivo (Dozois y Westra, 2004). Esto pone de manifiesto la relevancia de incluir instrumentos de evaluación de la ansiedad en los programas dirigidos a la prevención indicada de la depresión.

Una limitación común a muchos de los trabajos analizados es que éstos no se fundamentan en un marco teórico de referencia (p.e., González et al., 2006; Zlotnick et al., 2001), lo que impide identificar los mecanismos subyacentes a los resultados de las intervenciones, y por tanto desarrollar programas de intervención eficaces y analizar la generalización de sus resultados (Vázquez y Torres, 2007). Además, esto conlleva la ausencia de estudios en los que se hayan evaluado posibles mediadores. Algunos trabajos han encontrado una reducción de la sintomatología depresiva que ocurría concurrentemente con mejorías en variables cognitivas (Allart-van Dam et al., 2003, 2007; Gillham et al., 2006; Konnert et al., 2009; Seligman et al., 1999, 2007; Van Voorhees et al., 2008, 2009); incremento en el nivel de actividades agradables (Allart-van Dam et al., 2003, 2007); o el aumento de la autoestima (Allart-van Dam et al., 2003, 2007). Pero sólo en un estudio se encontró un papel mediador claro de algunas de esas variables. Allart-van Dam et al. (2003) encontraron que los cambios en las cogniciones depresivas y autoestima actuaban como mediadores de los cambios en los síntomas depresivos, mientras que no se encontraron efectos de mediación para las actividades agradables. Por otra parte, Stice et al. (2006) encontraron resultados interesantes en este sentido; comparando distintos grupos de intervención con un grupo control no tratamiento, los datos evidenciaron que el grupo de intervención cognitivo-conductual no conseguía mejores resultados que el grupo de apoyo expresivo, lo que indicaría que las habilidades cognitivo-conductuales (p.e., la reducción de las cogniciones negativas y el incremento de las actividades agradables) podrían no ser necesarias para producir reducciones significativas en los síntomas; pero que además, el hecho de que la intervención cognitivoconductual no consiguiese mayores efectos que la biblioterapia, escritura expresiva o escribir un diario, indicaría que ni los procedimientos cognitivo-conductuales, ni los factores inespecíficos asociados a la terapia de grupo, son necesarios para reducir la depresión, sino que hay múltiples maneras de reducirla. Stice et al. (2006) hipotetizaron que uno de los mecanismos a través de los que procedimientos tan diferentes funcionan es el incremento de la autoeficacia. Sería interesante realizar futuras investigaciones que incluyan un instrumento específico para valorar la autoeficacia, y ver si efectivamente podría estar actuando como una variable mediadora de los resultados.

La mayoría de los programas incluyen intervenciones complejas, que requieren personal altamente especializado para su implementación, lo que dificulta el acceso universal a estos recursos, por la escasez de profesionales disponibles. Los datos señalados apoyan la importancia de desarrollar y evaluar intervenciones preventivas eficaces, que sigan un modelo de prevención indicada, y que incluyan estrategias más sencillas. Además, muchos de esos trabajos presentan importantes limitaciones metodológicas, que restringen el alcance de los resultados. Dependiendo del estudio concreto, estas limitaciones metodológicas tienen que ver con tamaños muestrales pequeños (p.e., Lynch et al., 1997; Zlotnick et al., 2001); con la ausencia de ocultamiento de la asignación (p.e., Seligman et al., 1999) y de enmascaramiento (p.e., Clarke et al., 1995; Willemse et al., 2004); con no utilizar tratamientos específicos, replicables y recogidos en manuales de interven- 
ción (p.e., Garber et al., 2009; Sheffield et al., 2006); con la ausencia de información sobre la adherencia al tratamiento (p.e., Arnarson y Craighead, 2009; González et al., 2006); con el escaso empleo de medidas multimodales para evaluar los resultados (p.e., Van Voorhees et al., 2008, 2009); no realizar ciego de los evaluadores (p.e., Clarke et al., 2001; Konnert et al., 2009); y, con la ausencia de medidas de seguimiento (p.e., Lynch et al., 1997). Asimismo, se necesitan estudios que demuestren un mayor coste-efectividad de los ensayos de prevención indicada, frente a los del tratamiento tradicional y otro tipo de estrategias preventivas. Sólo Lynch et al. (2005) analizaron el coste-efectividad empleando la muestra de Clarke et al. (2001), informando de unos resultados comparables del programa preventivo con otros tratamientos aceptables para la depresión, evitando además importantísimos costes personales y familiares derivados del padecimiento de sintomatología depresiva grave continuada, y las posibles consecuencias derivadas de la misma (p.e., mayor riesgo de suicidio).

\section{Referencias}

Allart-van Dam, E., Hosman, C. M. H., Hoogduin, C. A. L. y Schaap, C. P. D. R. (2003). The coping with depression course: Short-term outcomes and mediating effects of a randomized controlled trial in the treatment of subclinical depression. Behavior Therapy, 34, 381-396. doi:10.1016/S0005-7894(03)80007-2

Allart-van Dam, E., Hosman, C. M. H., Hoogduin, C.A.L. y Schaap, C. P. D. R. (2007). Prevention of depression in subclinically depressed adults: Follow-up effects on the 'Coping with Depression' course. Journal of Affective Disorders, 97, 219-228. doi:10.1016/j.jad.2006.06.020

Arnarson. E. O. y Craighead, W. E. (2009). Prevention of depression among Icelandic adolescents. Behaviour Research and Therapy, 47, 577-585. doi: 10.1016/j.brat.2009.03.011

Arnarson. E. O. y Craighead, W. E. (2011). Prevention of depression among Icelandic adolescents: A 12-month follow-up. Behaviour Research and Therapy, 49, 170-174. doi: 10.1016/j.brat.2010.12.008

Butler, A. C., Chapman, J. E., Forman, E. M. y Beck, A. T. (2006). The empirical status of cognitive-behavioral therapy: A review of meta-analyses. Clinical Psychology Review, 26, 17-31. doi:10.1016/j.cpr.2005.07.003

Calear, A. L. y Christensen, H. (2010). Systematic review of school-based prevention and early intervention programs for depression. Journal of Adolescence, 33, 429-438. doi: 10.1016/j.adolescence.2009.07.004.

Clarke, G. N., Hawkins, E., Murphy, M.., Sheeber, L. B., Lewinsohn, P. M. y Seeley, J. R. (1995). Targeted prevention of unipolar depressive disorder in an at-risk sample of high school adolescents: A randomized trial of a group cognitive intervention. Journal of the American Academy of Child and Adolescent Psychiatry, 34, 312-321. doi:10.1097/00004583-19950300000016

Clarke, G., Hornbrook, M. C, Lynch, F. L., Polen, M., Gale, J., Beardslee, W. R., ... Seeley, J.R. (2001). A randomized trial of a group cognitive intervention for preventing depression in adolescent offspring of depressed parents. Archives of General Psychiatry, 58, 1127-1134.doi: 10.1001 /archpsyc. 28.12.1127

Cohen, J. (1988). Statistical power analysis for the behavioural sciences ( $\left.2^{\mathrm{a}} \mathrm{ed}.\right)$. Hillsdale, New Jersey: Lawrence Erlbaum Associates.

Dobson, K. S., Hopkins, J. A., Fata, L., Scherrer, M. y Allan, L. C. (2010). The prevention of depression and anxiety in a sample of high-risk adolescents: A randomized controlled trial. Canadian Journal of School Psychology, 25, 291-310. doi: 10.1177/0829573510386449

Dozeman, E., van Marwijk, H. W. J., van Schaik, D. J. F., Smit, F., Stek, M. L., van der Host, H. E., ...Beekman, A. T. F. (2012). Contradictory effects for prevention of depression and anxiety in residents in homes for
En conclusión, a pesar de la evidencia de que los programas de prevención indicada pueden reducir los síntomas de depresión en distintas poblaciones, pocos han demostrado que la incidencia de la depresión puede ser reducida, y que los cambios logrados tengan significación clínica. Urge la realización de ensayos adicionales con un adecuado rigor metodológico para estudiar el impacto de la prevención en la aparición de la depresión, así como el paso de los participantes en las intervenciones de un estado disfuncional a uno funcional. También es necesario evaluar las variables mediadoras del cambio y el coste-efectividad de estas intervenciones. Además, dado que sólo tres de los estudios de la revisión se realizaron en España, es necesario realizar más investigaciones de este tipo en nuestro medio.

Agradecimientos.-Este trabajo fue financiado por el proyecto PGIDT05PXIA21101PR de la Dirección Xeral de Investigación e Desenvolvemento (Consellería de Innovación, Industria e Comercio) de Galicia (España).

the elderly: A pragmatic randomized controlled trial. International Psychogeriatrics, 24, 1242-1251. doi: 10.1017/S1041610212000178.

Dozois, D. J. A. y Westra, H. A. (2004). The nature of anxiety and depression: Implications for prevention. In D. J. A. Dozois y K. S. Dobson (Eds.), The prevention of anxiety and depression: Theory, research and practice (pp.73-98). Washington, DC: American Psychological Association.

Fledderus, M., Bohlmeijer, E. T., Pieterse, M. E. y Schreurs, K. M. G. (2012). Acceptance and commitment therapy as guided self-help for psychological distress and positive mental health: A randomized controlled trial. Psychological Medicine, 42, 485-495. doi: 10.1017/S0033291711001206

Forsman, A. K., Schierenbeck I., y Wahlbeck, K. (2011). Psychosocial interventions for the prevention of depression in older adults: systematic review and meta-analysis. Journal of Aging and Health, 23, 387-416. doi: $10.1177 / 0898264310378041$.

Gabilondo, A., Rojas-Farreras, S., Vilagut, G., Haro, J. M., Fernández, A. Pinto-Meza, A. y Alonso, J. (2010). Epidemiology of major depressive episode in a southern European country: Results from the ESEMeDSpain project. Journal of Affective Disorders, 120, 76-85. doi: 10.1016/j.jad.2009.04.016

Garber, J., Clarke, G. N., Weersing, V. R., Beardslee, W. R., Brent, D. A. Gladstone, T.G.R.,...Iyengar, S. (2009). Prevention of depression in atrisk adolescents. Journal of the American Medical Association, 301, 22152224. doi: $10.1001 /$ jama.2009.788

Gillham, J. E., Hamilton, J., Freres, D. R., Patton, K. y Gallop, R. (2006). Preventing depression among early adolescents in the primary care setting: A randomized controlled study of the Penn Resiliency Program. Journal of Abnormal Child Psychology, 34, 203-219. doi: 10.1007/s10802005-9014-7

González, S., Fernández, C., Pérez, J. y Amigo, I. (2006). Prevención secundaria de la depresión en atención primaria. Psicothema, 18, 471-477.

Grupo de Trabajo sobre el Manejo de la Depresión Mayor en el Adulto. (2008). Guía de Práctica Clínica sobre el Manejo de la Depresión Mayor en el Adulto. Madrid: Plan Nacional para el SNS del MSC. Axencia de Avaliación de Tecnoloxías Sanitarias de Galicia (avalia-t). Guías de Práctica Clínica en el SNS: avalia-t $\mathrm{N}^{\mathrm{o}} 2006 / 06$

Higgins, J. y Green, S. (2008). Cochrane handbook for systematic reviews of interventions. Hoboken NJ: John Wiley \& Sons.

Horowitz, J. L. y Garber, J. (2006). The prevention of depressive symptoms in children and adolescents: A meta-analytic review. Journal of Consulting and Clinical Psychology, 74, 401-415. doi: 10.1037/0022-006X.74.3.401 
Jané-Llopis, E., Hosman, C., Jenkins, R. y Anderson, P. (2003). Predictors of efficacy in depression prevention programmes. Meta-analysis. The British Journal of Psychiatry, 183, 384-397. doi: 10.1192/bjp.183.5.384

Konnert, C., Dobson, K. y Stelmach, L. (2009). The prevention of depression in nursing home residents: A randomized clinical trial of cognitivebehavioral therapy. Aging \& Mental Health, 13, 288-299. doi: 10.1080/13607860802380672

Korte, J., Bohlmeijer, P., Cappeliez, P., Smit, F., y Westerhof, G. J. (2012). Life review therapy for older adults with moderate depressive symptomatology: A pragmatic randomized controlled trial. Psychological Medicine, 42, 1163-1173. doi: 10.1017/S0033291711002042.

Lara, M. A., Navarro, C. y Navarrete, L. (2010). Outcome results of a psycho-educational intervention in pregnancy to prevent PPD: A randomized controlled trial. Journal of Affective Disorders, 122, 109-117. doi:10.1016/j.jad.2009.06.024

Le, H. N., Perry, D. F. y Stuart, E.A. (2011). Randomized controlled trial of a preventive intervention for perinatal depression in high-risk Latinas. Journal of Consulting and Clinical Psychology, 79, 135-141. doi: $10.1037 / \mathrm{a} 0022492$

Lewinsohn, P. M. y Clarke, G. N. (1984). Group treatment of depressed individuals: The "Coping With Depression" course. Advances in Behaviour Research and Therapy, 6, 99-114. doi: 10.1016/0146-6402(84)90005-5

Liu, E. T. H., Chen, W. L., Li, Y. H., Wang, C. H., Mok, T.J. y Huang, H. S. (2009). Exploring the efficacy of cognitive bibliotherapy and a potencial mechanism of change in the treatment of depressive symptoms among the Chinese: A randomized controlled trial. Cognitive Therapy and Research, 33, 449-461. doi: 10.1007/s10608-008-9228-4

Lynch, D. J., Tamburrino, M. B. y Nagel, R. (1997). Telephone counseling for patients with minor depression: Preliminary findings in a family practice setting. The Journal of Family Practice, 44, 293-298.

Mathers, C. D. y Loncar, D. (2006). Projections of global mortality and burden of disease from 2002 to 2030. PLOS MEDICINE, 3, e442. doi: 10.1371/journal.pmed.0030442

Merry, S., McDowell, Hetrick, S., Bir, J. y Muller, N. (2008). Psychological and/or educational interventions for the prevention of depression in children and adolescents [Cochrane Review]. En The Cochrane Library, 4. Chichester, UK: John Wiley \& Sons. doi: 10.1002/14651858.CD003380.pub2

Mossey, J. M., Knott, K. A., Higgins, M. y Talerico, K. (1996). Effectiveness of a psychosocial intervention, interpersonal counseling for subdysthymic depression in medically ill elderly. Journal of Gerontology: Medical Sciences, 51A, M172-M178. doi: 10.1093/gerona/51A.4.M172

Mrazek, P. y Haggerty, R.J. (1994). Reducing risks for mental disorders. Frontiers for preventive intervention research. Washington, DC: National Academy Press.

Muñoz, R. F., Le, H. N., Ghosh Ippen, C., Díaz, M. A., Urizar, G. G., Soto, J.,...Lieberman, A.F. (2007). Prevention of postpartum depression in low-income women. Development of the Mamás y Bebés/Mothers and Babies course. Cognitive and Behavioral Practice, 14, 70-83. doi: 10.1016/j.cbpra.2006.04.021

National Institute for Clinical Excellence (2009). Depression: the treatment and management of depression in adults. Obtenido de http:/ /www.nice.org.uk/nicemedia/live/12329/45888/45888.pdf

Oei, T.P.S. y Dingle, G. (2008). The effectiveness of group cognitive behaviour therapy for unipolar depressive disorders. Journal of Affective Disorders, 107, 5-21. doi: 10.1016/j.jad.2007.07.018

Organización Mundial de la Salud. (2004). Prevention and treatment of mental disorders. Effective interventions and policy options. Summary report. Ginebra: Autor.

Puskar, K., Sereika, S. y Tutsaie-Mumford, K. (2003). Effect of Teaching Kids to Cope (TKCC) Program on outcomes of depression and coping among rural adolescents. Journal of Child and Adolescent Psychiatric Nursing, 16, 71-80. doi: 10.1111/j.1744-6171.2003.tb00350.x

Roberts, C., Kane, R., Bishop, B., Matthews, H. y Thomson, H. (2004). The prevention of depressive symptoms in rural school children: A follow up study. International Journal of Mental Health Promotion, 6, 4-16. doi: 10.1080/14623730.2004.9721934.

Roberts, C., Kane, R., Thomson, H., Bishop, B. y Hart, B. (2003). The prevention of depressive symptoms in rural school children: A randomized controlled trial. Journal of Consulting and Clinical Psychology, 71, 622-628. doi: 10.1037/0022-006X.71.3.622

Seligman, M. E. P., Schulman, P., DeRubeis, R. J. y Hollon, S. D. (1999). The prevention of depression and anxiety. Prevention and Treatment, 2, Article $8 . \quad$ Obtenido de http://journals.apa.org/prevention/volume2/pre0020008a.html. doi: 1037/1522-3736.2.1.28a

Seligman, M. E. P., Schulman, P. y Tryon, A.M. (2007). Group prevention of depression and anxiety symptoms. Behavior Research and Therapy, 45, 1111-1126. doi: 10.1016/j.brat.2006.09.010

Sheffield, J. K., Spence, S. H., Rapee, R. M., Kowalenko, N., Wignall, A., Davis, A. y McLoone, J. (2006). Evaluation of universal, indicated, and combined cognitive-behavioral approaches to the prevention of depression among adolescents. Journal of Consulting and Clinical Psychology, 74, 66-79. doi: 10.1037/0022-006X.74.1.66

Spence, S., Sheffield, J., y Donovan, C. (2003). Preventing adolescent depression: An evaluation of the Problem Solving for Life Program. Journal of Consulting and Clinical Psychology, 71, 3-13. doi: 10.1037/0022006X.71.1.3

Stamp, G. E., Williams, A. S. y Crowther, C. A. (1995). Evaluation of antenatal and postnatal support to overcome postnatal depression. A randomized controlled trial. Birth, 22, 138-143. doi: 10.1111/j.1523536X.1995.tb00689.x

Stice, E., Burton, E., Bearman, S. K. y Rohde, P. (2006). Randomized trial of a brief depression prevention program: An elusive search for a psychosocial placebo control condition. Behavior Research and Therapy, 45, 863876. doi:10.1016/j.brat.2006.08.008

Stice, E., Rohde, P., Gau, J. M. y Wade, E. (2010). Efficacy trial of a brief cognitive-behavioral depression prevention program for high-risk adolescents: Effects at 1- and 2-year follow-up. Journal of Consulting and Clinical Psychology, 78, 856-867. doi: 10.1037/a0020544

Stice, E., Rohde, P., Seeley, J. R. y Gau, J.M. (2008). Brief cognitivebehavioral depression prevention program for high-risk adolescents outperforms two alternative interventions: A randomized efficacy trial. Journal of Consulting and Clinical Psychology, 76, 595-606. doi: $10.1037 / \mathrm{a} 0012645$

Valladares, A., Dilla, T. y Sacristán, J.A. (2009). La depresión: una hipoteca social. Últimos avances en el conocimiento del coste de la enfermedad. Actas Españolas de Psiquiatría, 37, 49-53.

Van Voorhees, B. W., Fogel, J., Reinecke, M. A., Gladstone, T., Stuart, S., Gollan, J.,...Bell, C. (2009). Randomized clinical trial of an internetbased depression prevention program for adolescents (Project CATCHIT) in primary care: 12-week outcomes. Journal of Developmental and Behavioral Pediatrics, 30, 23-37. doi: 10.1097/DBP.0b013e3181966c2a

Van Voorhees, B.W., Vandeprough-Booth, K., Fogel, J., Gladstone, T., Bell, C., Stuart, S.,...Reinecke, M. (2008). Integrative internet-based depression prevention for adolescents: A randomized clinical trial in primary care for vulnerability and protective factors. Journal of the Canadian Academy of Child and Adolescent Psychiatry, 17, 184-196.

Van't Veer-Tazelaar, N., Van Marwijk, H., Van Oppen, P., Van Hout, H., Van der Horst. H. E., Cuijpers, P.,...Beekman, A. T. (2009). Steppedcare prevention of anxiety and depression in late life. Archives of General Psychiatry, 66, 297-304. doi: 10.1001/archgenpsychiatry.2008.555

Van't Veer-Tazelaar, N., Van Marwijk, H., Van Oppen, P., Van der Horst. H. E., Smit, F., Cuijpers, P.,...Beekman, A.T. (2011). Prevention of late-life anxiety and depression has sustained effects over 24 months: A pragmatic randomized trial. American Journal of Geriatric Psychiatry, 19, 230-239. doi: 10.1097/JGP.0b013e3181faee4d

Vázquez, F. L., Otero, P., Torres, A., Hermida, E., Blanco, V., y Díaz, O. (2013). A brief problem-solving indicated-prevention intervention for prevention of depression in nonprofessional caregivers. Psicothema, 25, 87-92. doi: 10.7334/psicothema2012.89

Vázquez, F. L., Torres, A., Blanco, V., Díaz, O., Otero, P., y Hermida, E. (2012). Comparison of relaxation training with a cognitive-behavioural intervention for indicated prevention of depression in university students: A randomized controlled trial. Journal of Psychiatric Research, 46, 1456-1463. doi: 10.1016/j.jpsychires.2012.08.007.

Vázquez, F. L. y Torres, A. (2007). Análisis sobre la investigación de la prevención de episodios nuevos de depresión. Clínica y Salud, 18, 221-246. 
Westerhof, G. J., Bohlmeijer, E. T., van Beljouw, I. M. J. y Pot, A.M. (2010). Improvement in personal meaning mediates the effects of a life review intervention on depressive symptoms in a randomized controlled trial. The Gerontologist, 50, 541-549. doi: 10.1093/geront/gnp168

Willemse, G. R. W. M., Smit, F., Cuijpers, P. y Tiemens, B.G. (2004). Minimal-contact psychotherapy for sub-threshold depression in primary care. British Journal of Psychiatry, 185, 416-421. doi: 10.1192/bjp.185.5.416.

Young, J. F., Mufson, L. y Davies, M. (2006). Efficacy of Interpersonal Psychotherapy-Adolescent Skills Training: An indicated preventive inter- vention for depression. Journal of Child Psychology and Psychiatry, 47, 1254 1262. doi: 10.1111/j.1469-7610.2006.01667.x

Young, J. F., Mufson, L. y Gallop, R. (2010). Preventing depression: A randomized trial of interpersonal psychotherapy-adolescent skills training. Depression and Anxiety, 27, 426-433. doi: 10.1002/da.20664

Zlotnick, C., Johnson, S. L., Miller, I. W., Pearlstein, T. y Howard, M. (2001). Postpartum depression in women receiving public assistance: Pilot study of an interpersonal-therapy-oriented group intervention. American Journal of Psychiatry, 158, 638-640.

(Artículo recibido: 03/11/2011; revisado: 03-05-2013; aceptado: 16-05-2013) 